\title{
2D Material Membranes for Operando Atmospheric Pressure Photoelectron Spectroscopy
}

\author{
Robert S. Weatherup ${ }^{1,2}$
}

Published online: 1 November 2018

(c) The Author(s) 2018

\begin{abstract}
Probing the chemistry that occurs at catalyst interfaces under realistic process conditions is key to the rational design of better materials for industrial catalytic reactions. Ambient pressure X-ray photoelectron spectroscopy, has until recently been limited to pressures two orders of magnitude below atmosphere. However, the development of photoelectron transparent membranes based on two-dimensional materials that can maintain large pressure differences and yet have thicknesses approaching or even falling below the inelastic mean free path of photoelectrons, now allows the atmospheric pressure regime and above to be accessed. We introduce here the fundamental principles underlying this membrane-based approach to atmospheric pressure photoelectron spectroscopy, and in this context highlight some of the key design concepts and challenges in performing experiments with this technique. We discuss a number of recent proof-of-concept studies, and highlight the potential of the membrane-based approach for operando characterisation of catalyst interfaces under reaction conditions, as well as some current challenges and limitations in this area.
\end{abstract}

Keywords Graphene $\cdot$ Atmospheric pressure $\cdot$ XPS · Operando spectroscopy $\cdot$ Reaction cell

\section{Introduction}

Heterogeneous catalysis is essential for the synthesis and purification of industrial chemicals at the scale demanded by modern society, and in mitigating the impact of harmful pollutants on health and the environment by converting them to more inert products [1]. Heterogeneous catalytic reactions per definition take place at the interfaces between different phases, most commonly a solid catalyst with reactants in either the gas or liquid phase. Probing and understanding the chemistry of such interfaces under realistic process conditions is key to the rational design of better catalyst materials. Photoelectron spectroscopy can provide element specific information on the chemical state of a catalyst surface, with nanometre-scale surface sensitivity, as a result of the small inelastic mean free-paths $(\lambda)$ of low-energy electrons in solids. This limited $\lambda$ however also places severe

Robert S. Weatherup

robert.weatherup@manchester.ac.uk

1 University of Manchester at Harwell, Diamond Light Source, Didcot, Oxfordshire OX11 0DE, UK

2 School of Chemistry, University of Manchester, Oxford Road, Manchester M13 9PL, UK constraints on the measurement environment, meaning high vacuum conditions have traditionally been required to ensure the effective inelastic mean free path of the photoelectrons exceeds their trajectories through the spectrometer. Siegbahn et al. partially addressed this issue by the using a differentially pumped aperture, that establishes a pressure gradient between the sample surface and the electron analyser, effectively increasing $\lambda$ and allowing gas phase measurements up to 1 mbar [2]. The advent of commercially available near ambient pressure X-ray photoelectron spectroscopy (NAPXPS) systems has further increased this limit into the tens of mbar regime [3], with multiple differentially pumped apertures used to achieve a large pressure drop between the sample and spectrometer, whilst electrostatic lenses focus the photoelectrons through the apertures to maintain the collection efficiency [4]. Nevertheless, many heterogeneously catalysed reactions of industrial relevance occur at atmospheric pressures and above, or indeed in liquid environments. The importance of achieving these realistic reaction conditions can be understood by considering the change in chemical potential, $\Delta \mu=\mathrm{kT} \cdot \ln \left(\mathrm{p} / \mathrm{p}^{0}\right)$, of an adsorbate in equilibrium with a gas, as the pressure, $p$, is increased whilst at a given temperature, T. For a reaction occurring at $200^{\circ} \mathrm{C}$, this 'pressure driving force' corresponds to $\mathrm{a} \sim 1.1 \mathrm{eV}$ difference 
between atmospheric pressure and ultra high vacuum $\left(10^{-9}\right.$ mbar), and is still $\sim 0.2 \mathrm{eV}$ when compared to the pressures routinely achieved with existing NAP-XPS systems (10 mbar). This can alter which chemical phases and surface structures are thermodynamically stable, as well as lift certain kinetic limitations and thereby change the reaction pathways. In order to obtain a detailed understanding of the underlying mechanisms involved in such reactions, spectroscopic methods are needed that can probe interfaces at these higher pressures.

When investigating solid-liquid interfaces, extracting photoelectrons presents an even greater challenge, given the even higher densities of liquid and solid phases, which are typically three orders of magnitude greater than atmospheric pressure gases [5]. Therefore to ensure that a significant proportion of photoelectrons can escape from the interface and be detected by the spectrometer, the thickness of either the liquid or the solid should ideally be below the corresponding $\lambda$. One successful approach to this has been the "dip \& pull" method where a planar electrode is dipped into a beaker of solution and then retracted by several millimetres to form a thin liquid film of a few tens of nanometres in thickness on the surface of the electrode $[6,7]$. Tender X-ray excitation allows probing of the solid-liquid interface, as the photoelectrons produced have sufficiently high kinetic energies that a significant proportion pass through the liquid film without being inelastically scattered and can then be collected by a NAP-XPS analyser. Maintaining such a meniscus of stable thickness over the course of hours needed for in situ measurements is however far from straightforward, requiring control over the equilibrium vapour pressure to avoid changes in the solute concentration, and potentially cooling of the liquid to reduce its vapour pressure and avoid excessive photoelectron attenuation by gas-phase scattering. Concerns also remain over whether the constrained transport of ions in this nanometer-thick liquid film is representative of real electrochemical systems. The use of tender X-rays also sacrifices surface sensitivity compared to soft XPS, and the lower photoionisation cross-sections of core levels may mean a brighter $\mathrm{X}$-ray source or longer acquisition times are necessary.

An alternative approach is to instead reduce the thickness of the solid such that photoelectrons are able to pass through, and then use this to enclose a high pressure reaction cell that can be filled with either gas or liquids, whilst the rest of the measurement chamber and analyser remain at much lower pressures. This is achieved by using an electron transparent membrane that maintains the pressure difference and, given that $\lambda$ for the solid membrane remains unaffected by the environment within the reaction cell, allows greater freedom over the environment within the reaction cell without adversely affecting the collection of photoelectrons from the solid-gas or solid-liquid interface.
This membrane-based approach has already been widely applied for environmental cell transmission electron microscopy (EC-TEM), and X-ray absorption spectroscopy (XAS) using thin $(\sim 10-200 \mathrm{~nm})$ silicon nitride membranes that are transparent to high energy electrons, and X-rays [8-10]. XAS is able to provide element-specific chemical information with surface-sensitivity by acquisition of the total electron yield signal, including under operando conditions [11-13]. However depth-profiling has so far been limited to blunt comparisons between the surfacesensitive total electron yield signal (defined by $\lambda$ ), and the more bulk-sensitive total fluorescent yield signal (defined by the X-ray attenuation length-typically two-orders of magnitude larger than $\lambda$ ). Furthermore, access to a synchrotron light source is required to provide a bright source of tuneable X-ray energy. Even then the time-resolution is often limited to several minutes due to dead-time associated with moving and stabilizing mechanical components as the X-ray energy is scanned [14], although this can be at least partially addressed by continuous scanning of the monochromator (and undulator if used) with on-the-fly data collection [15].

By comparison, XPS using soft X-ray excitation allows probing of the whole range of core levels with binding energies below the excitation energy, even when a fixed energy laboratory X-ray source is used. The limited value of $\lambda$ ensures nanometre-scale interface sensitivity, and depth-profiling can be achieved by tuning the excitation energy, which in turn alters the kinetic energy and thus $\lambda$ of the photoelectrons (see Fig. 1a), or in the case of a laboratory source, by tilting the sample to vary the photoelectron escape angle and thus escape depth. A further advantage is that XPS data is often more readily interpretable than XAS, with interpretation of the latter often requiring simulation of the acquired spectra [11].

In order to perform XPS using the membrane-based approach, an extremely thin membrane of similar thickness to $\lambda$ is required so that sufficient photoelectrons are transmitted without inelastic scattering. The focus of this article is therefore the recent development of such membrane-based approaches using two-dimensional (2D) material membranes to perform soft XPS at atmospheric pressures and above. The fundamental principles underlying this technique are introduced, along with the practical considerations in performing such measurements. A number of recent studies that demonstrate the potential of this technique are discussed. This approach is not without challenges, particularly in the preparation of the membrane materials and the deposition of active catalyst or electrode materials, and these are critically discussed along with possible pathways to overcome some of the existing limitations of this technique. 

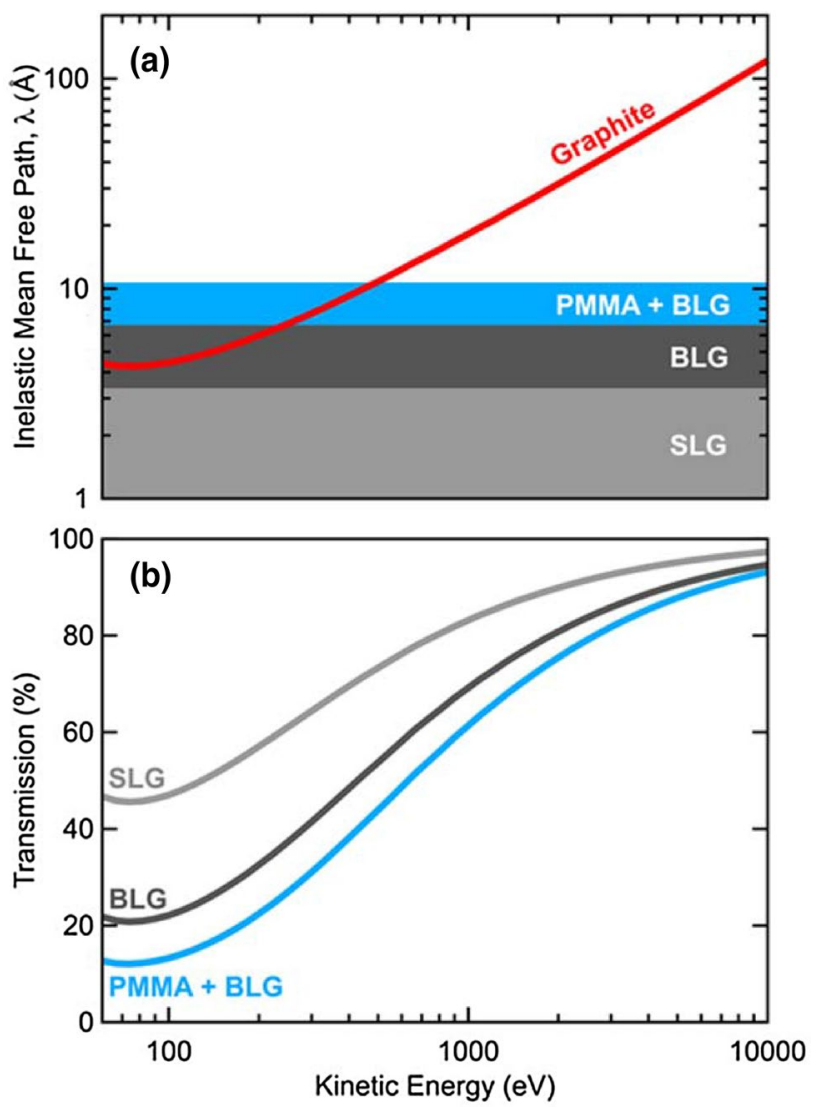

Fig. 1 a Variation in the inelastic mean free path, $\lambda$, of electrons in graphite with kinetic energy. Shaded regions indicate the thicknesses of a single layer of graphene (SLG), two layers of graphene (BLG), and two layers of graphene covered with polymethylmethacrylate (PMMA) residues from graphene transfer (PMMA+BLG). The PMMA residues are considered as a continuous film of $0.4 \mathrm{~nm}$ thickness [16]. b Corresponding plots of electron transmission for SLG, BLG, and PMMA + BLG, considering only inelastic scattering. Values of $\lambda$ for Graphite [17] and PMMA [18] are obtained from optical data and interpolation using the modified Bethe equation

\section{Photoelectron Transparent Membranes}

The membrane used to enclose a reaction cell must be impermeable and mechanically robust enough to maintain the large pressure drop from the high pressure gas or liquid environment within the cell to the vacuum conditions of the measurement chamber. At the same time it must be electron transparent enough that a significant portion of the photoelectrons generated on the high pressure side can pass through without being inelastically scattered. The inelastic mean free path of electrons in a solid, $\lambda$, provides a measure of this transparency to photoelectrons. Compared to the case where no membrane is present, $\sim 37 \%$ of the photoelectrons are expected to pass through a membrane of thickness $\lambda$ without being inelastically scattered, reducing to only $\sim 5 \%$ for a membrane of $3 \lambda$ thickness. Values of $\lambda$ typically vary within a relatively narrow range for different solids, giving rise to the somewhat crude approximation of a "universal curve" for the variation of $\lambda$ with electron energy $[19,20]$. There is thus little option to increase $\lambda$ by selection of the membrane material, and so to maximise the detectable photoelectron signal either the value of $\lambda$ must be increased by adjusting the photoelectron energy, or the membrane thickness must be reduced. Given the photoelectrons detected during XPS typically have kinetic energies exceeding $100 \mathrm{eV}$, Fig. 1a shows that in this range $\lambda$ will monotonically increase with photoelectron kinetic energy i.e. with increasing the X-ray excitation energy. This approach of increasing $\lambda$ was used in some of the early membrane-based XPS experiments, where hard X-rays $(\sim 6000 \mathrm{eV})$ were used in combination with $\sim 15 \mathrm{~nm}$ thick Si membranes to observe electrochemical oxidation by measuring the Si $2 \mathrm{p}$ peak $\left(\lambda_{\mathrm{Si}} \approx 10 \mathrm{~nm}\right)$ [21]. However this also increases the inelastic mean free path of photoelectrons in the reaction cell environment, and thus comes at the expense of reduced surface sensitivity which limits its usefulness in resolving surface species. Furthermore the photoionisation cross-section of a given atomic subshell reduces dramatically as the X-ray excitation energy is increased into the hard X-ray regime [22], meaning either a much higher photon flux or longer acquisition time is required.

Soft X-rays are therefore generally preferred for photoelectron spectroscopy, and so in the context of the membrane-based approach, the membrane thickness must be reduced to below a few nanometers. Many materials can not be readily produced at such thicknesses, or are not stable as a freestanding membrane, agglomerating into larger particles to minimise surface energy. However, 2D materials can remain stable as single-layer sheets thanks to their strong in-plane bonding, even when only a single atom thick as in the case of graphene and hexagonal boron-nitride $(\mathrm{hBN})$. Although the precise thickness of a 2D material is ill-defined, the interlayer spacing of $\sim 0.34 \mathrm{~nm}$ in graphite is a reasonable working value for graphene [23]. Figure 1a therefore shows how $\lambda_{\text {graphite }}$ varies with electron energy [17], with the thicknesses of single and bilayer graphene (S-/BLG) indicated for reference.

Graphene, the archetypal 2D material, is particularly appealing for the membrane-based approach to atmospheric pressure XPS. The impermeability of pristine graphene to gases including $\mathrm{He}$ [24], has led to significant interest in its use as a passivating barrier to prevent, for example, the oxidation of metal surfaces [25-29]. However in realistic use, the presence of atomic defects and grain boundaries typically results in some permeability to both gases and ions [30-33]. The strong in-plane bonding of graphene yields impressive mechanical robustness, with the breaking strength of pristine graphene, exceeding all other materials so far measured [34]. Although this is also reduced somewhat by the presence of lattice defects and grain boundaries [35-37], relatively 
defective graphene sheets can still be stable as free-standing membranes and sustain large pressure differences [24, 38, 39]. Graphene further offers electrical conductivity helping to avoid charging during X-ray illumination [40], as well as serving as a current path for electrochemical control during measurements. It is also relatively chemically inert, given its position at the top of the electrochemical series [41] and the absence of out-of-plane covalent bonds, which contributes to the stability of the membranes under reaction conditions and $\mathrm{X}$-ray illumination. Other 2D materials such as $\mathrm{h}-\mathrm{BN}$, the electrically insulating counterpart to graphene, offer complementary properties such as improved thermal stability [42], that may be desirable when a high-temperature or oxidising reaction environment is to be studied. Therefore, the recent emergence of methods to controllably produce these $2 \mathrm{D}$ materials over relatively large-areas has made the fabrication of sub-nanometre thickness membranes for atmospheric pressure XPS a realistic prospect, as will be discussed in the next section.

\section{2D Membrane Fabrication}

\subsection{Graphene Growth}

In order to realise an impermeable and mechanically stable membrane that is transparent to photoelectrons, a continuous covalently bonded graphene layer is desired over an area comparable to the $\mathrm{X}$-ray spot size (typically tens to hundreds of micrometre). As already highlighted this should ideally be uniform SLG so that the transmission of photoelectrons is maximised. A variety of methods are now available for producing graphene and other 2D materials, that vary widely in the quality, uniformity, and cost of the material produced. The highest quality graphene material, i.e. with fewest defects and grain boundaries, remains that mechanically exfoliated from highly-oriented pyrolytic graphite (HOPG) crystals by, for example, peeling with scotch tape [43]. However, samples have to be individually screened to find the relatively small single-layer graphene flakes amongst the flakes of varying thickness that are produced, making this process labour intensive and impractical for all but "heroic" device fabrication. Liquid-phase exfoliation attempts to automate the splitting of graphite by using sonication or shear mixing with suitable solvents or dispersing agents to produce graphene suspensions at significantly lower cost [44, 45]. Alternatively, hydrophobic graphite can be chemically converted to make hydrophilic materials such as graphene oxide, allowing aqueous suspensions without the need for dispersants [46]. Percolated films can then be relatively easily produced, and in the case of graphene oxide can subsequently be (at least partially) reduced [47]. However these films consist of networks of micrometre-sized platelets that are not covalently bonded together, making them much less impermeable and mechanically stable than pristine graphene.

Much higher-quality material can be achieved over large-areas by the thermal decomposition of hexagonal $\mathrm{SiC}$ crystals, by heating to $>1000{ }^{\circ} \mathrm{C}[48,49]$. This is relatively expensive given the high thermal budget and costs in producing the $\mathrm{SiC}$ wafers. Furthermore the difficulty in detaching the graphene from the $\mathrm{SiC}$ substrate has been a major barrier to its use as a material for membranes, which although possible typically leads to the creation of rips and tears unless carefully optimised [50, 51].

Chemical vapour deposition (CVD) has therefore emerged as the preferred technique for producing highquality, SLG over large areas for many applications [52, 53]. This involves exposing a catalyst support, typically a transition metal foil/film to a gaseous/vapor carbon precursor at elevated temperatures. The precursor dissociation supplies carbon to the catalyst surface that diffuses into the catalyst bulk until a carbon supersaturation develops (locally) at the catalyst surface, resulting in the nucleation of graphene domains that grow with continuing precursor exposure until they coalesce and knit together to form a continuous covalently bonded network [54]. Growth therefore depends on the balance between the supply and removal of carbon from the catalyst surface as summarised in Fig. 2a.

Although catalytic formation of SLG was initially demonstrated on expensive single-crystalline supports prepared under ultra-high-vacuum conditions [56, 57], a remarkable aspect of graphene CVD is that high-quality, continuous sheets of only a single-atom in thickness can be grown conformally over relatively rough, low-cost, polycrystalline catalyst foils [55]. This includes the rolling striations induced by the manufacturing process, topography associated with the catalysts microstructure such as grain boundaries, as well as surface roughening resulting from restructuring and sublimation at the elevated growth temperatures.

$\mathrm{Cu}$ foils were the first low-cost supports on which uniform SLG was grown [58], and have become the most widely used catalyst supports for CVD thanks to the relatively broad range of processing conditions over which SLG can be stabilised. Initially, this was widely attributed to the low $\mathrm{C}$ solubility of $\mathrm{Cu}$ with a single-layer graphene assumed to form at high temperature during hydrocarbon exposure [58, 59], whilst the growth of multilayer graphene seen on higher solubility catalysts such as $\mathrm{Ni}$ was assumed to result from the precipitation of carbon due to the reduction in solubility on cooling [60-62]. This was in part based on a comparison between growth on $25 \mu \mathrm{m}$ thick $\mathrm{Cu}$ foils and $\mathrm{Ni}$ thin films of only several hundred nanometres deposited onto $\mathrm{SiO}_{2}$ [59], but subsequent in situ experiments have shown such a comparison to be misleading [63,64]. As illustrated in Fig. $2 b$, thin films are quickly saturated with carbon given 

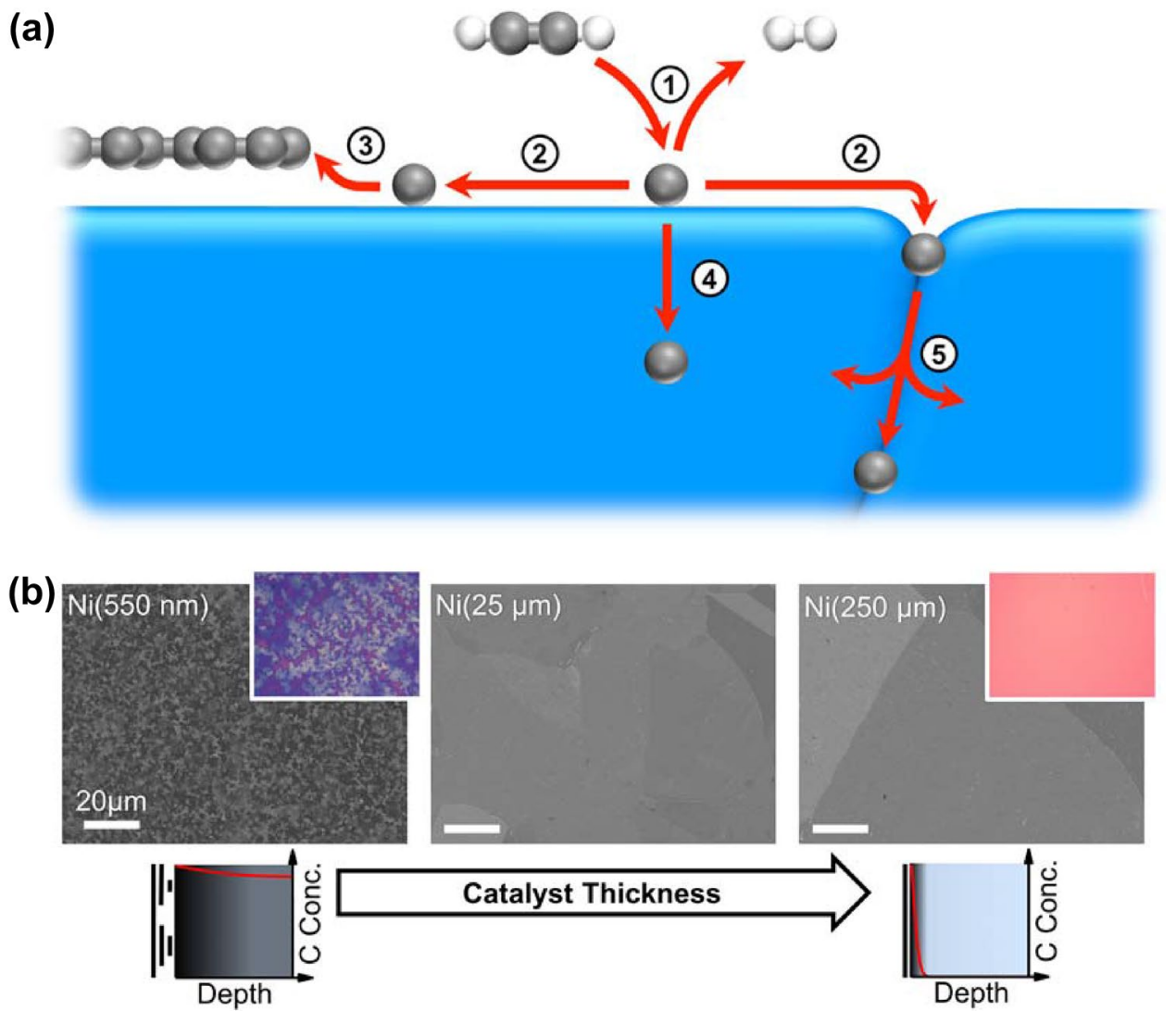

Fig. 2 a Key processes involved in the CVD of graphene on a polycrystalline catalyst. Process 1: precursor dissociation supplies carbon to the catalyst surface. Process 2: surface diffusion transports carbon across the catalyst surface. Process 3: carbon attaches to the edge of a growing graphene domain. Process 4: carbon diffusion into the bulk of the catalyst removes carbon from the surface. Process 5: grain boundary diffusion serves as a more-rapid pathway for carbon removal from the surface. Adapted with permission from

the smaller extent of their bulk [33, 65], leading to inhomogeneous multi-layer graphene formation occurring at temperature during hydrocarbon exposure. For thicker catalyst foils the supply of carbon to the catalyst surface continues to be mediated by diffusion into the catalyst bulk and grain boundaries, enabling uniform SLG formation as has now been demonstrated on a variety of suitably thick transition metal foils [33, 65-67]. This includes catalysts with a relatively high $\mathrm{C}$ solubility e.g. $\mathrm{Co}, \mathrm{Ni}, \mathrm{Pt}$, with in situ experiments confirming that SLG growth occurs predominantly isothermally via the process described earlier, with the majority of dissolved $\mathrm{C}$ quenched within the catalyst bulk for typical experimental cooling rates [64]. Nevertheless, $\mathrm{Cu}$ remains the preferred catalyst for many applications particularly when the graphene is to be transferred to another substrate (see below), in part due to the ease with which it can be etched with relatively benign chemicals, but also because it doesn't contain a significant amount of carbon
[55]. Copyright 2016 American Chemical Society. b Scanning electron micrographs of elemental $\mathrm{Ni}$ catalysts of increasing thicknesses $(550 \mathrm{~nm}, 25 \mu \mathrm{m}$, and $250 \mu \mathrm{m})$, following $\mathrm{C}_{2} \mathrm{H}_{2}\left(10^{-5} \mathrm{mbar}\right)$ exposure at $\sim 600{ }^{\circ} \mathrm{C}$ for $15 \mathrm{~min}$. All scale bars are $20 \mu \mathrm{m}$. Insets: corresponding optical images of the graphene films transferred to $\mathrm{SiO}_{2}(300 \mathrm{~nm}) /$ $\mathrm{Si}$, shown at the same scale. Adapted with permission from [33]. Copyright 2012 American Chemical Society

dissolved within its bulk, that can otherwise remain as a source of contamination after etching [33].

Regardless of the catalyst used, the nucleation of graphene at multiple points on the catalyst surface results in a graphene layer that is polycrystalline, and thus contains numerous grain boundary defects that may make the graphene less leak-tight. In order to reduce the density of grain boundaries, higher growth temperatures can be used to yield larger domain sizes, as well as lower defect densities within domains, due to the increased $\mathrm{C}$ diffusivity that favours attachment to existing domains rather than secondary nucleation [68]. Further reductions in nucleation density can be achieved by passivating high-reactivity surface sites such as step edges to reduce the density of preferred nucleation sites $[33,63,69,70]$, or by providing a low density of even more preferred nucleation sites or seeds [71, 72], or supplying the precursor locally [73], and these approaches can now yield $\mathrm{cm}$-sized domains. Alternatively, grain boundaries can 
in principle be avoided by nucleating multiple epitaxiallyaligned graphene domains on a single crystalline substrate, that stitch together as they impinge on one another. This has been demonstrated on relatively low-cost, wafer-scale substrates that are potentially re-usable, such as Ge(110) [74] and annealed polycrystalline $\mathrm{Cu}$ foils that have been converted to $\mathrm{Cu}(111)$ [75]. Whether there are still defects associated with the merging of these aligned domains to form this pseudo-single crystalline graphene remains a subject of ongoing research.

Given the potentially high-quality material that can be produced, and the relatively low-barriers to entry, for both the graphene growth and transfer process (see below), CVD seems likely to remain the de facto choice for producing graphene and other 2D materials for photoelectron transparent membranes.

\subsection{Graphene Transfer}

In order to use graphene as a membrane it must be suspended over a suitable support, which requires transfer from the catalyst foil used for growth. In spite of its impressive breaking strength [36], unsupported graphene is prone to tearing and wrinkling and thus a support-layer is typically used to facilitate transfer. Figure $3 \mathrm{a}$ outlines the general procedure used, in which the upper surface of the graphene covered catalyst foil is first coated with the support layer (e.g. polymer), the catalyst foil is then removed by wet etching, or other methods such as electrochemical bubbling [66, 76]. The supported graphene is then floated on deionized water to remove etchant residues, lifted out onto the surface of the target substrate, and then left to dry. Finally the support is dissolved away using a suitable solvent (e.g. acetone) or etchant, to leave graphene covering the support. However, this transfer method has been found to result in significant contamination of the graphene films, associated with incomplete removal of the polymer support. Atomic force microscopy (AFM) has revealed polymer residues of average thickness of $\sim 1 \mathrm{~nm}$ can cover $\sim 40 \%$ of the graphene surface even after rinsing in acetone and isopropanol. Figure $1 \mathrm{~b}$ shows how the presence of these polymer residues will significantly reduce the electron transmission of graphene membranes. Although numerous approaches have been proposed to remove these residues, these are often accompanied by degradation of the graphene, as in the case of annealing treatments which show poor selectivity towards polymer-removal, or simply convert the polymer to amorphous carbon [77, 78]. Polymer-free transfer approaches have therefore been developed that completely avoid the use of a support that covers the graphene surface $[55,79,80]$. Figure $3 b$ details one such approach where rather than coating with a polymer, a support frame made from self-adhesive aluminium foil is attached around the sample edges. On etching of the catalyst foil, this frame provides mechanical support to avoid significant wrinkling

\section{(a) POLYMER-SUPPORTED TRANSFER}

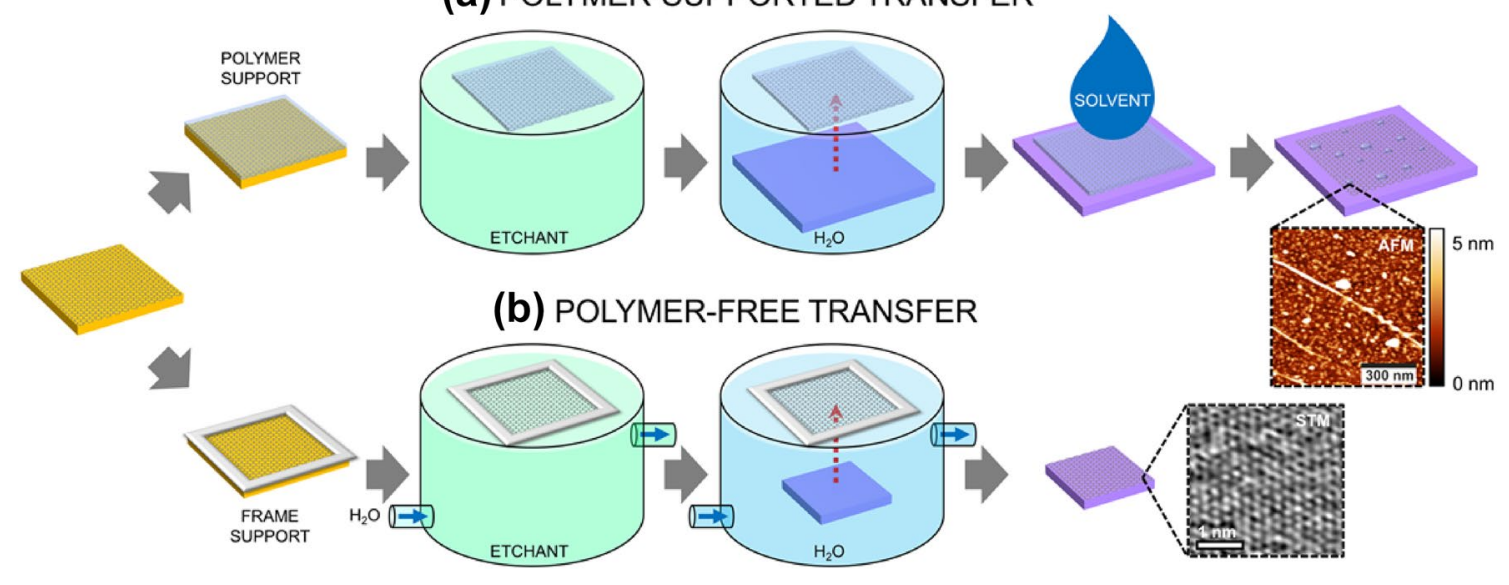

Fig. 3 Graphene transfer methods. a Polymer-supported graphene transfer, where graphene on a metal foil is coated with a thin polymer layer, placed on the surface of an etchant solution to remove the metal, then rinsed in water and lifted out onto a desired substrate. After drying, the polymer support is then removed using an appropriate solvent, however this typically leaves significant polymer residues. Inset: atomic force micrograph of graphene transferred onto a $\mathrm{SiO}_{2} / \mathrm{Si}$ wafer using a PMMA-supported method without any post-treatment, where small bright features are visible which correspond to PMMA residues with a relative coverage of $\sim 40 \%$ and average thickness of
$1 \mathrm{~nm}$. Inset: reprinted from [16], with the permission of AIP Publishing. b Polymer-free transfer, where a self-adhesive frame around the edges of the sample supports the graphene during etching of the metal foil whilst leaving the central region uncovered. The etchant is gently flushed away with the frame-supported graphene still floating on the liquid surface, and then the graphene is lifted out onto the surface of the substrate and left to dry. Inset: scanning tunnelling micrograph of suspended graphene transferred by the polymer-free method without any additional post-treatment, showing the graphene lattice with atomic resolution $\left(\mathrm{V}_{\mathrm{S}}=0.18 \mathrm{~V}, \mathrm{I}_{\mathrm{t}}=500 \mathrm{pA}, 2 \mathrm{D}\right.$-FFT filtered) 
and tearing whilst leaving the central region of graphene free-standing and thus uncontaminated by support materials. The etchant is then carefully rinsed away by slowly flushing with water whilst the frame-supported graphene remains floating on the surface, and then the target substrate is lifted through the centre of the frame to pick up the free-standing graphene and left to dry. The cleanliness of this polymer-free transfer-process has been confirmed by scanning tunnelling microscopy (STM), which reveals the hexagonal graphene lattice with atomic resolution [38]. We note that comparable STM characterization is generally not possible for graphene contaminated with polymer, as when scanning over these non-conducting regions the STM tip would either crash or become coated with the polymer.

Whether a polymer support is used or not, transfer that involves a wet etching step often induces mechanical damage, associated with the surface tension of the liquid. Indeed the physisorption of airborne contaminants such as hydrocarbons renders many substrates more hydrophobic following extended air exposure [81, 82]. The increased contact angle means that after the graphene is lifted out, it does not lie flat on the substrate during drying leading to cracking and wrinkling [83]. Cracking can obviously result in the membrane leaking, whilst wrinkling will reduce photoelectron transmission and the adhesion of the graphene to the underlying substrate, as well as inducing high local stress making these regions more prone to chemical attack. This situation can be improved by making the support more hydrophilic with treatments such as $\mathrm{O}_{2}$ plasma, ultraviolet/ozone cleaning, or annealing [81, 84-86], that remove contamination and/or chemically activate the surface, or by using lower surface energy solvents [83]. Alternatively dry transfer processes have been demonstrated that avoid liquids altogether by peeling the graphene from the surface of suitably weakly interacting metals (see Sect. 7) [87-89]. As well as avoiding the damage associated with wet processing, this eliminates contamination related to residual etchants, although the supports used (e.g. polymers) can still leave residues.

Irrespective of the transfer method, the inherent roughness of the polycrystalline foils typically used as growth support will nevertheless result in some wrinkling of the transferred graphene as it relaxes onto an often much flatter membrane support. This includes morphology related to the foil manufacturing process (e.g. rolling striations), as well as grain growth and catalyst sublimation at the elevated growth temperature, with the latter particularly affecting $\mathrm{Cu}$ catalyst foils whose growth temperature is often close to the melting point of $\mathrm{Cu}[68,90]$. It may be possible to reduce this wrinkling by for example using specially prepared flat substrates [91], and growth conditions that minimise roughening [68]. In addition, wrinkling may already occur prior to transfer during cooling of the growth substrate [92, 93], as a result of the different thermal expansion coefficients of the catalyst and graphene. The use of a support substrate can preserve these wrinkles following transfer, even if the strain that caused their formation is released on removal of the graphene film. Therefore care is needed in selection of the catalyst material and its texture to avoid such wrinkling in the first place $[94,95]$.

The reliable fabrication of leak-free membranes remains one of the most significant challenges in applying the membrane-based approach to atmospheric pressure photoelectron spectroscopy using 2D materials, in most part due to the shortcomings of the transfer process. In future, it would therefore be desirable to eliminate the transfer step by directly forming free-standing graphene membranes. One possible route is to fabricate suspended catalyst thin-films on perforated $\mathrm{Si}_{3} \mathrm{~N}_{4}$ supports using established microelectromechanical systems (MEMS) techniques, then cover the catalyst surface with graphene using CVD, and finally etch away the catalyst to leave suspended graphene. This would require optimisation of the catalyst film thickness and material, and most-likely a low-temperature growth process to avoid pinhole formation [63, 70], but could potentially avoid the mechanical damage and polymer residues associated with existing transfer techniques.

\section{Reaction Cell Designs}

A variety of reaction cell designs have been developed for performing membrane-based atmospheric pressure XPS, with the cell geometry influencing the choice of support substrate for the suspended graphene membranes, the measurement setups that can be used, and the systems that can be measured. Figure 4 summarises several of the major approaches. The first demonstrations of XPS of a liquid through a graphene oxide layer were performed using a single-aperture reaction cell (Fig. 4a) [96]. In this case, sealing only a single, micrometre-scale aperture with graphene is achieved much more reliably than sealing a larger hole, or array of holes, covering a much large area. However, the relatively small "active" area for measurement, means that a measurement setup with a highly focussed X-ray beam is required, limiting this approach to micro-focussed X-ray sources. The high X-ray flux-density required to achieve a sufficient signal intensity from such sources has the potential to induce significant radiolysis effects [13, 135]. Indeed, the detection of water vapour during the early measurements with this geometry is attributable to the radiolysis of the liquid water contained within the cell as a result of X-ray illumination $[96,97]$. Therefore Kolmakov et al. introduced an alternative approach of using an array of microchannels which are covered with graphene on one end (working electrode), filled with a liquid electrolyte, and with the other end capped with an adhesive sealant that if conductive (e.g. 

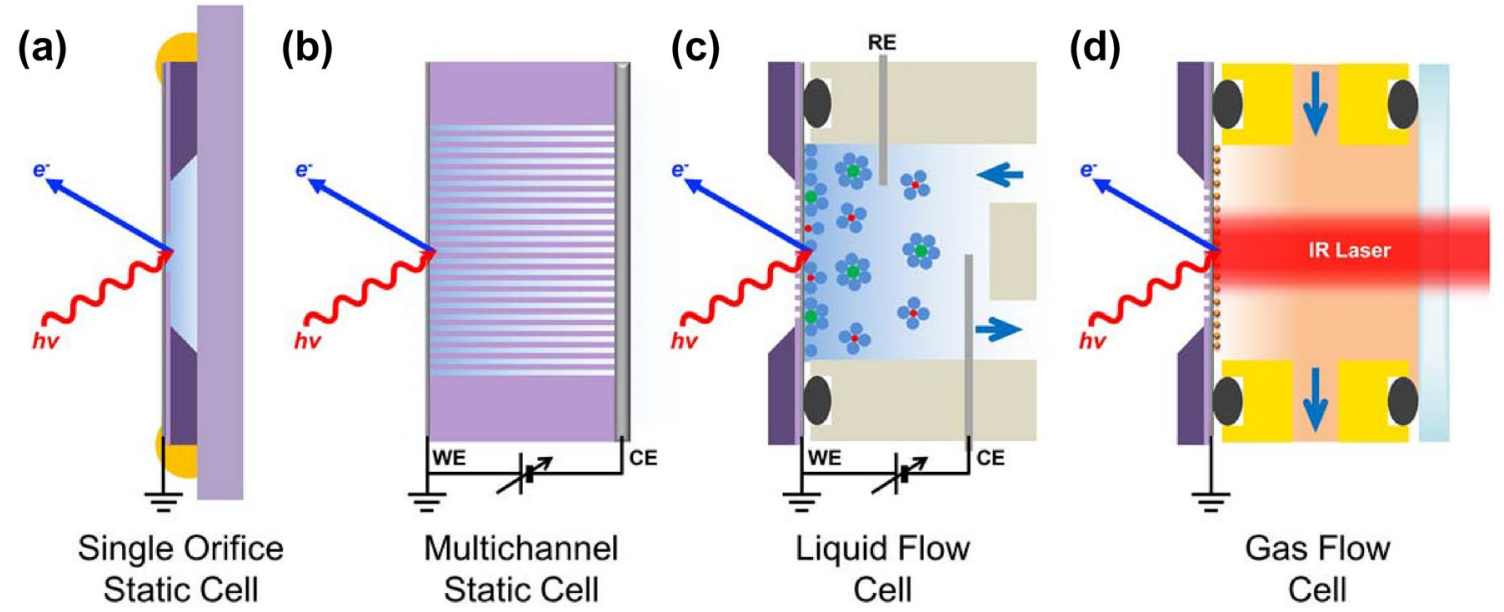

Fig. 4 Schematics illustrating different graphene-sealed reaction cell geometries. a Single orifice static cell, b multichannel static cell, $\mathbf{c}$ liquid flow cell, $\mathbf{d}$ gas flow cell

solidified Ga) can also serve as the counter electrode [97, 98]. Given that the size of an individual graphene covered aperture is limited by the area over which graphene can be reliably suspended (typically of the order of micrometres), this array of holes provides a larger total active area for measurement meaning that a less-focussed X-ray beam can be used whilst still detecting a sufficient photoelectron signal. This is particularly important when measuring dense phases such as atmospheric pressure gases or liquids, where the effects of radiolysis can become significant [13], and the local build-up of radiolysis products is exacerbated by a higher X-ray flux density. Furthermore, given that each microchannel forms a separate liquid cell this approach can significantly improve the reliability of these cells for measurement: even if several of the cells are not properly sealed or burst during measurement, the other intact cells can continue to be measured, with the minuscule liquid volumes released unlikely to cause problems for the measurement chamber's vacuum system. However, if multiple cells are illuminated, the acquired spectra will combine contributions from both burst and unburst cells, whose relative proportions may vary over time. Therefore a means of obtaining spatial resolution (e.g. photoemission electron microscopy) may be needed to separate these different contributions [98]. Even with imaging, correlating the electrochemical measurement which will combine contributions from all of these cells, which may have leaked to varying extents, with the measured photoelectron signal may be a complex task.

The small volume of the individual cells and the fact they are static also imposes limitations on the measurements that can be performed with this approach, which means that in their current form they are not well-suited to studying solid-gas catalytic reactions. In particular, there is no opportunity to perform a pretreatment or regeneration step to yield a desired catalyst state prior to introducing the reactants, as the environment within the cells can not be readily exchanged. This also means the reaction must be performed in a batch mode, rather than flow mode, with the timescale over which the reaction can proceed limited by the small quantity of reactants within each cell. This is clearly less of a problem when studying electrochemical reactions with liquid electrolytes within the cell, as for many reactions of interest the electrochemical cycle can be repeated numerous times, and the sweep rate varied as required. The other potential complication is that the process for sealing the cells requires careful consideration to ensure it is compatible with the environment to be trapped within them, so that it neither dissolves away causing leakage, nor reacts with the cell environment which might completely change the behaviour observed.

An alternative approach that ensures a larger active area, but avoids numerous, enclosed static volumes, is to use a thin perforated support such as an $\mathrm{Si}_{3} \mathrm{~N}_{4}$ membrane containing numerous holes covered with graphene which enclose a single, larger reaction chamber (Fig. 4c, d) [38, $99,100]$. Clearly this does not afford the same improvement in reliability as the static multi-channel arrays, as a single leak will affect the whole volume behind the membrane. Whilst this would present a major problem when measuring liquid environments within the cell as the liquid volume could leak out to cover the whole measurement area, when measuring in an atmospheric pressure gas environment several leaks can be tolerated as the small flow of gas can be easily pumped away by the analysis chamber pumps, whilst a continuous flow of gas into the reaction cell maintains the pressure at the desired level [38]. Sealing of the reaction cell with these perforated supports can be easily achieved using O-rings 
(black in Fig. 4c, d), whose material can be selected to ensure compatibility with the measurement conditions, with various temperature and chemically resistant polymers available.

The ability to change or refresh the reaction atmosphere allows much more flexibility in the measurements that can be performed for both liquid- and gas-phase reaction environments. This is particularly advantageous for studying solid-gas interfaces where the catalyst can be pre-treated and the reaction operated in flow mode with reactants continuously supplied. Figure $4 d$ shows one such measurement scheme for this, where catalyst nanoparticles can be deposited onto the high-pressure side of the graphene membrane, and can be directly heated by laser illumination through an optical window.

\section{Detection Threshold}

Having discussed different supports that may be used to create stable 2D material membranes, it is important to understand the concentration of species that can be effectively detected when performing atmospheric pressure XPS measurements with the membrane-based approach. Although the detection threshold will depend on a number of factors including the X-ray source, elements being probed, and length of acquisition, as an approximate rule of thumb the detection threshold with standard XPS can be taken as $\sim 0.1$ at.\%. For the membrane-based approach we must consider the reduced photoelectron transmission through the graphene, as well as the porosity of the perforated support used, as a significant portion of the area illuminated by the X-ray beam will not be suspended graphene. At present, the commercially available $\mathrm{Si}_{3} \mathrm{~N}_{4}$ supports with the highest active area have a hexagonal close packed pattern with a pitch that is double the hole diameter, corresponding to a porosity, and thus active area, of $\sim 23 \%$. More typically, however, a porosity of $15-20 \%$ is used to increase stability of the $\mathrm{Si}_{3} \mathrm{~N}_{4}$ support. Considering the detection of photoelectrons with a kinetic energies of $\sim 600 \mathrm{eV}$, this would suggest that for a SLG membrane the detection threshold would be $\sim 0.6$ at. $\%$, rising to $\sim 1$ at.\% for PMMA-transferred BLG (see Fig. 1b). For aqueous solutions this corresponds to concentrations of $\sim 350 \mathrm{mM}$, and $\sim 550 \mathrm{mM}$ respectively. In order to further improve this detection threshold, the porosity of the membrane support may be increased but this will ultimately be limited by the corresponding reduction in its mechanical stability, leaving few further options beyond using the thinnest and cleanest possible graphene layer.

\section{Electrode/Catalyst Preparation}

As mentioned earlier graphene has the advantage of being relatively chemically inert in its pristine form, making it ideal as a model catalyst support [101, 102]. In order to study catalytic reactions, methods are needed to deposit suitable catalyst materials onto this support. Given the limited $\lambda$ for photoelectrons generated by soft X-rays, the thickness of the catalyst/electrode being probed is critical, particularly if the intention is to probe the solid-liquid or solid-gas interface. One approach is to deposit dispersed nanoparticles onto the graphene membrane, such that there will still be a reasonable proportion of uncovered membrane surface in contact with the reaction environment. This will allow the collection of photoelectrons originating from the surfaces of the catalyst nanoparticles in contact with graphene, those in contact with the reaction environment, as well as the reaction environment itself. Alternatively, very thin continuous films can be deposited that uniformly cover the graphene surface. Such uniform model surfaces are less realistic catalyst materials, but are more amenable for interpreting depth-resolved measurements, as the complexities associated with the nanoparticle coverage and contributions from the sides of the nanoparticles are avoided. 2D materials are clearly ideal for this purpose and the formation of intimate heterostructures between many 2D materials has already received extensive research interest for various electronic applications [103]. The formation of continuous oxide films on the surface of graphene, that are below a nanometre in thickness have also been well-demonstrated [26, 104, 105]. Given graphene's absence of out-of-plane covalent bonds, many materials are liable to agglomerate on its surface particularly when at elevated temperatures. One solution to this might be to functionalise the graphene membrane, however such functionalisation is likely to negatively impact upon its mechanical and chemical stability.

Irrespective of the exact morphology of the deposited catalyst materials, the method of depositing them should be carefully selected to avoid severely damaging the graphene membrane. Energetic methods such as sputtering or pulse laser deposition should be avoided as these are likely to induce defects in the graphene. Less energetic deposition methods such as evaporation, atomic layer deposition, electrochemical/electroless deposition, or even drop casting are therefore generally preferable. Although these can all be performed after membrane fabrication, it may be advantageous to deposit these materials prior to any transfer processes to act as an additional support layer to help minimise any mechanical damage.

In fabricating the $2 \mathrm{D}$ material membranes materials with core levels that will interfere with those of interest in 
the reaction should obviously be avoided where possible. Although the choices for membrane support may be limited to materials that can be produced with the required dimensions and strength, these can easily be coated with thin layers of different materials using physical vapor deposition methods in order to eliminate contributions from the underlying material. In this same regard, it is also important to avoid the use of materials that may catalyse the attack of the 2D material under reaction conditions $[106,107]$, which would otherwise undermine the stability of the membrane as discussed further below.

\section{Membrane Stability}

\subsection{Mechanical Stability}

Although CVD graphene is inherently polycrystalline, the well-stitched boundaries between differently oriented domains, retain the high breaking strength of graphene [36]. However, the presence of vacancy defects and wrinkling during the transfer process, are found to reduce the breaking strength (and young's modulus) [37]. The presence of defects therefore practically limits the size of suspended graphene regions to a few micrometres, when using commercially available SLG produced by CVD. Arrays of many small apertures covered with graphene are therefore preferred to improve the pressure stability as well as the transfer reliability, with the most common supports currently being perforated $\mathrm{Si}_{3} \mathrm{~N}_{4}$ membranes, or multichannel-arrays made from mineral-glass.

Given graphene's lack of out-of-plane covalent bonds, the layers in graphite are only held together by weak van-derWaal's-like bonding, facilitating mechanical exfoliation. The adhesion of the graphene to its support must therefore also be carefully considered, as if this is not strong enough the environment within the reaction cell will be able to bypass the graphene membrane and leak out along the graphenesupport. Metallic films are therefore commonly deposited onto these supports prior to graphene transfer to try and improve this adhesion, as well as minimise charging by making the support more conductive [38, 108]. The intercalation of species beneath 2D materials has been studied on a range of transition metal supports [29, 107, 109, 110], with a strong interaction between a 2D material and its support shown to be critical to preventing intercalation [29]. A simple distinction can be drawn between strongly and weakly interacting substrates based on the extent to which the band structure of the 2D material is altered. For a weakly interacting substrate, the band structure is left largely intact, which in the case of graphene means the characteristic linear dispersion at the $\mathrm{K}$ point is retained with only the Fermi-level being altered due to charge transfer from the substrate, as seen for $\mathrm{Cu}$ [111-113], Ag [111], Ir [114], Pt [57, 115, 116], and $\mathrm{Au}[120,111,117]$. For more strongly interacting metals, e.g. Ni [57, 118-121], Co [122, 123], Fe [121, 124], the characteristic linear dispersion is lost due to hybridization between the graphene $\pi$ and metal $d$ valence band states. Therefore although Au coated supports have been used in several of the reports on graphene membranes for atmospheric pressure XPS, we note that other metal coatings may offer superior adhesion, yielding membranes that are more leak-tight and potentially stable for longer periods. However, given the wet processing typically involved in graphene transfer and possible plasma treatment of the support, an idealised interface between a clean, metallic surface is not obtained. Therefore an annealing process following transfer may be necessary to clean the interface and activate any strong interaction.

\subsection{Chemical Stability}

The chemical stability of the membranes is also a key consideration. At room temperature, pristine graphene is relatively chemically inert given its position at the top of the electrochemical series [41], and the absence of out-of-plane covalent bonds. However, grain boundaries and vacancy defects in CVD graphene are more susceptible to chemical attack. Indeed, chemical treatments with aqueous solutions have been used to create nanopores of controlled size in CVD graphene to achieve selective ion transport $[31,125,126]$. This is also observed in reactive gaseous environments, for example, in oxygen containing atmospheres at sufficiently high temperatures graphene starts to be burnt away [109], which again occurs preferentially at defects $[127,128]$ with damage induced at much lower temperatures and oxygen partial pressures than for pristine graphene/graphite [129]. As well as these more obvious routes to chemical attack, illumination of liquid and gases with ionising radiation such as X-rays and electrons can lead to the generation of reactive species by radiolysis $[130,131]$. This is particularly pronounced when low-energy (soft) X-rays/electrons are used to probe dense phases such as high pressure gases and liquids, as this radiation is absorbed in a relatively small volume close to the illuminated interface and diffusive transport of species away from the interface is relatively slow. This is highlighted in Fig. 5a, which shows the influence of electron beam illumination on a BLG membrane with deionised water sealed beneath it. After $<30$ s of illumination, multiple bright regions appear that grow in size with time and start to merge. These are attributed to bubbles formed by radiolysis beneath the graphene. After $\sim 68 \mathrm{~s}$ a brighter streak appears on the left-hand side of the graphene covered hole which may relate to etching/or damage to one of the graphene layers, and then after $\sim 73 \mathrm{~s}$ a tear through both graphene layers can be clearly seen in the bottom left-hand 


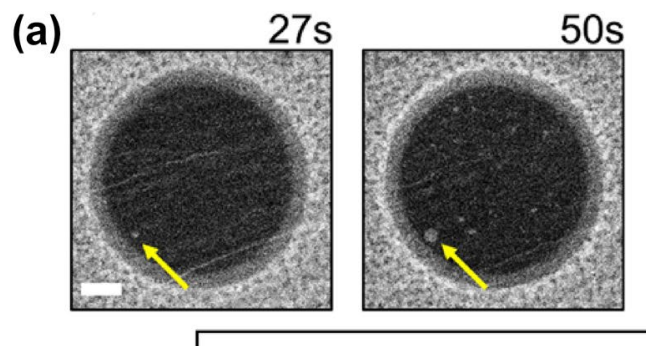

(b)

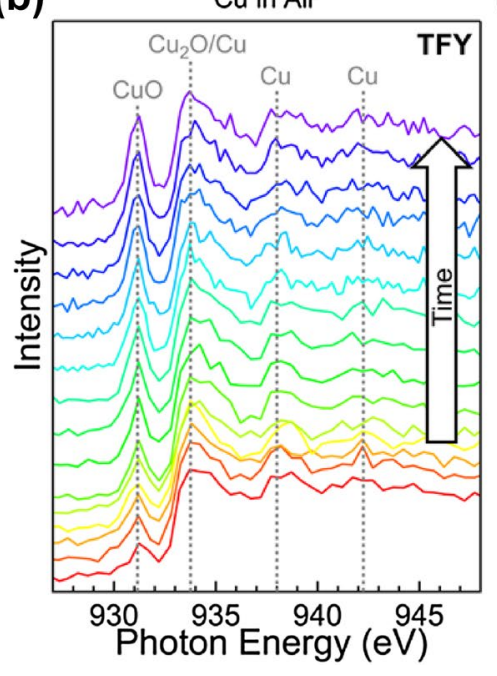

(c)

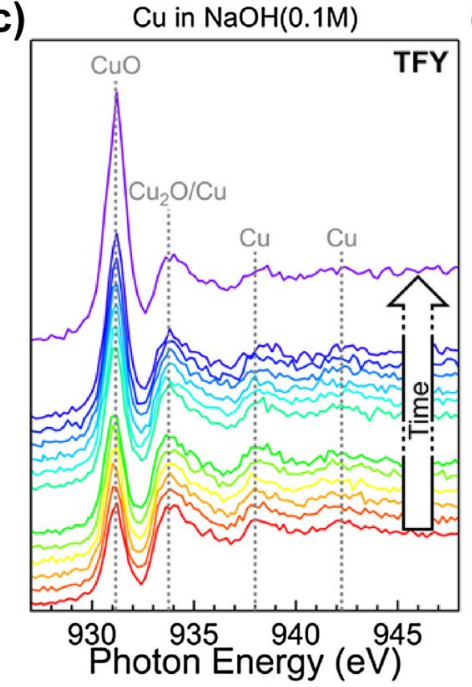

$60 \mathrm{~s}$

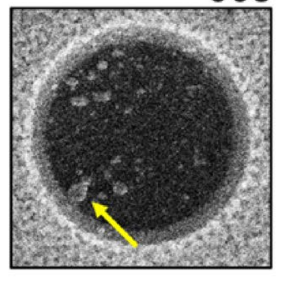

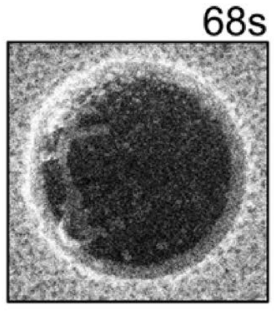

$68 s$

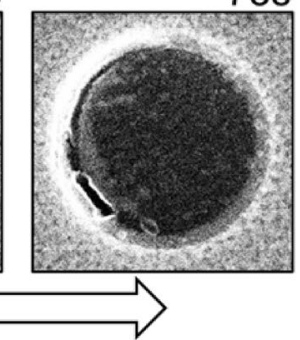

(d) $\mathrm{Cu}$ in $\mathrm{NaOH}(0.1 \mathrm{M})+\mathrm{CH}_{3} \mathrm{OH}(0.1 \mathrm{M})$

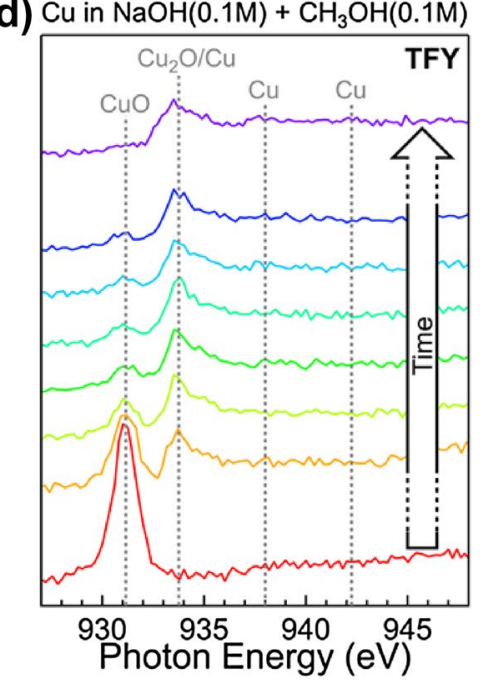

Fig. 5 a Series of scanning electron micrographs of a hole in a gold coated $\mathrm{Si}_{3} \mathrm{~N}_{4}$ support covered by 2-layers of graphene with liquid water sealed beneath. Images were acquired sequentially in the same region with an incident beam of $15 \mathrm{keV}$, using an Everhart-Thornley type secondary electron detector. Yellow arrows highlight a bubble that nucleates and grows beneath the membrane under continuous illumination with the electron beam, which after $\sim 70$ s leads to rupture of the membrane most likely due to chemical attack by radicals produced by water radiolysis. The scale bar is $200 \mathrm{~nm}$. Reproduced from [5] with permission from the PCCP Owner Societies. b-d Total fluorescent yield $\mathrm{X}$-ray absorption spectroscopy of the $\mathrm{Cu} \mathrm{L}_{3}$-edge

corner of the graphene covered hole, and many of the bubbles have now escaped. Given the lower mass density of gases, bubbles might be expected to have a lower secondary electron yield than the liquid and thus appear darker. Their bright contrast is however attributable to the higher secondary electron yield at edges, i.e. topographic contrast. Indeed, in several cases it is possible to discern brighter edges and darker centres to the bubbles, as also seen in other reports of bubble formation in water where secondary electron imaging is used [132]. Similar observations of bubble formation have also been made for aqueous solutions behind grapheneoxide membranes during illumination with a highly focussed X-ray beam [96]. The associated damage to the graphene may relate to the mechanical stress arising from surface tension at the bubble-water interface, or chemical attack by the radicals produced by radiolysis. This is further clarified by recent X-ray absorption measurements under atmospheric for a $\mathrm{Cu}(50 \mathrm{~nm})$ film measured (b) in air at atmospheric pressure [first six spectra acquired $\sim 150 \mathrm{~s}$ apart (red to green), with remaining spectra acquired $\sim 300 \mathrm{~s}$ apart (green to violet)], $\mathbf{c}$ after replacing air with an aqueous solution of $\mathrm{NaOH}(0.1 \mathrm{M})$, [acquired $\sim 400 \mathrm{~s}$ apart, except for $\sim 1300 \mathrm{~s}$ between the sixth and seventh spectra and $\sim 2200$ s between the penultimate and final spectra], $\mathbf{d}$ after changing to an aqueous solution of $\mathrm{NaOH}(0.1 \mathrm{M})$ and $\mathrm{CH}_{3} \mathrm{OH}(0.1 \mathrm{M})[\sim 360 \mathrm{~s}$ between the start of each spectrum acquisition, except for $\sim 3600 \mathrm{~s}$ between the first and second spectra and $\sim 2700$ s between the penultimate and final spectra.] Adapted with permission from [13]. Copyright 2018 American Chemical Society

pressure conditions, in both liquid and gas phase environments. Figure $5 b$ shows the evolution of the $\mathrm{Cu} \mathrm{L}_{3}$-edge during X-ray illumination of a $\mathrm{Cu}$ thin $(50 \mathrm{~nm})$ film in air, showing the gradual oxidation of the $\mathrm{Cu}$ surface towards $\mathrm{CuO}$, which is attributable to the local generation of ozone by radiolysis i.e. chemical attack. Figure $5 \mathrm{c}$ shows that this oxidation induced by X-ray illumination continues when the sample environment is changed to a basic aqueous solution, with the generation of hydroxyl radicals now chiefly implicated. This therefore suggests that chemical attack is responsible for the damage to graphene membranes seen in Fig. 5a, which is again likely to occur preferentially at defects.

Therefore improving the membrane's stability against chemical attack (including by the products of radiolysis) requires either the use of materials that are more resilient to chemical attack, or minimisation of the production of the chemical species responsible for attack. In the first case, 
using higher-quality graphene with fewer defects would reduce the number of sites for preferential attack, but this is practically limited by the quality of graphene that can be reliably obtained by CVD. Stacking multiple graphene layers may also be beneficial, as the probability of two defects overlapping will correspond to the square of the defect density of SLG, and this approach has indeed been used in several reports to improve stability [98, 99, 108, 133], although it comes at the expense of reduced photoelectron transmission, as discussed earlier. Instead of this, other 2D materials could be employed which are more resistant to oxidative attack such as h-BN [134], however its insulating behaviour is likely to result in undesirable charging during measurement. This may be surmountable, by, for example, stacking h-BN and graphene layers to achieve improved chemical resistance whilst maintaining sufficient conductivity. In the second case, the concentration of certain chemical species can be reduced by diluting the reaction environment with an inert solvent/gas, but this is also likely to alter the equilibrium of the reaction under investigation, leaving little further option for avoiding damage under the desired conditions. However, for the specific situation of chemical attack induced by the products of radiolysis, the concentration of these products can be relatively straightforwardly reduced by either lowering the X-ray/electron dose, or by spreading this over a larger area by using a less focussed beam or rapidly scanning the beam over the sample $[13,135]$. Another promising approach is to add scavenger species or "getters" which serve to provide decay routes for the damaging products of radiolysis to react to more inert products. Figure $5 \mathrm{~d}$ shows an example of this, where the addition of a small amount of methanol to the aqueous solution results in the reduction of $\mathrm{CuO}$ back to $\mathrm{Cu}_{2} \mathrm{O}$ over time with X-ray illumination. Such getters may be dissolved or suspended in a solution or placed in close proximity to the interface being probed [136], but care is needed in selecting these species to avoid altering the underlying chemistry that is being probed.

\section{Example Reactions}

\subsection{Solid-Liquid Interfaces}

As noted earlier, the first proof-of-concept demonstration of the using 2D material membranes to enable XPS of solid-liquid interfaces, used a single-aperture reaction cell covered with a relatively thick graphene oxide membrane, and filled with an aqueous NaI solution [96]. Liquid and vapor phase water was thereby observable in the $\mathrm{O} 1 \mathrm{~s}$ spectrum, however the signal associated with the respective ions dissolved in the solution was too weak to be detected. Velasco-velez et al. demonstrated a liquid flow reaction cell which was again filled with an aqueous solution, but this time of $\mathrm{CoSO}_{4}$ [99]. This was used to achieve electrodeposition onto the graphene membrane, with the emergence of a signal in the Co $2 p$ XPS region confirming the formation of an oxidised cobalt layer. This successfully demonstrated the ability to detect the chemical state of the deposited film through the graphene membrane, however it should be noted that the relatively thick layer deposited prevented probing of the ions in solution at the solid-liquid interface.

More recently, Kolmakov et al. have focussed on using multichannel static cells in combination with PEEM and other microscopic methods. This has included X-ray absorption measurements of the $\mathrm{O} \mathrm{K}$-edge of liquid water acquired in partial electron yield (PEY) mode [98], as well as similar Auger electron spectroscopy (AES) measurements [108]. This approach has also been applied to detect $\mathrm{Cu}$ ions in aqueous solutions of $\mathrm{CuSO}_{4}$ and $\mathrm{H}_{2} \mathrm{SO}_{4}$ during electrochemical cycling [133]. Measuring the $\mathrm{Cu} \mathrm{L}_{3}$-edge at different potentials reveals differences in the ratio of $\mathrm{Cu}^{+}$to $\mathrm{Cu}^{2+}$ ions as the graphene membranes is biased relative to a $\mathrm{Pt}$ counter electrode, consistent with copper(II) ion reduction on the graphene electrode. These results therefore highlight the promise of performing operando studies of solid-liquid interfaces under electrochemical control. These PEEM based studies have so far focussed on XAS measurements using PEY mode, which have an information depth of a few nanometres that is not easily varied. Ideally future measurements will be able to take advantage of a tuneable X-ray source when performing XPS, to vary the kinetic energy of photoelectrons and consequently their escape depths, and thus obtain depth-resolved chemical information about the species present at the solid-liquid interface during electrochemical reactions.

\subsection{Solid-Gas Interface}

The demonstration of atmospheric pressure XPS of gas phase reactions using graphene membranes has been a more recent development with the first reports emerging within the last 2 years, and thus the literature in this area remains relatively limited. The reports to date have all used flow cell designs of the type illustrated in Fig. 4d, which as noted earlier are more suited for studying reactions occurring at solid-gas interfaces. Weatherup et al. thereby used singlelayer graphene membranes produced with a polymer-free process to demonstrate simultaneous probing of the chemical state of catalyst nanoparticles and the reaction environment using XPS as illustrated in Fig. 6 [38].

Figure $6 \mathrm{~b}$ shows the O1s XP region, showing that with the introduction of $\mathrm{O}_{2}(1 \mathrm{bar})$ into the reaction cell, a double peak associated with gas phase oxygen can be detected, despite the graphene being partially covered with $\mathrm{Cu}$ nanoparticles. On introducing the $\mathrm{O}_{2}(1 \mathrm{bar})$, the $\mathrm{Cu}$ nanoparticles are observed to gradually oxidise towards $\mathrm{Cu}^{2+}$ 

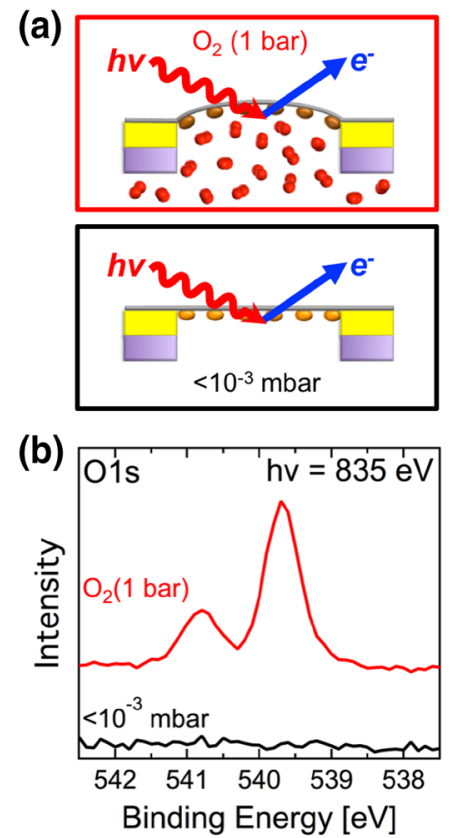

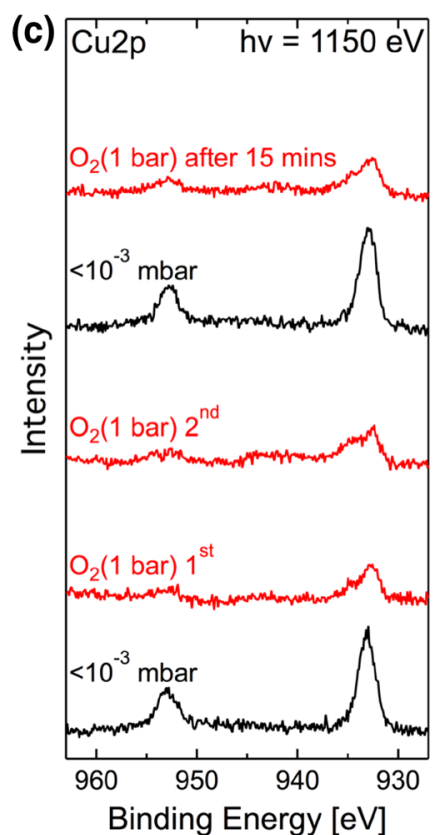

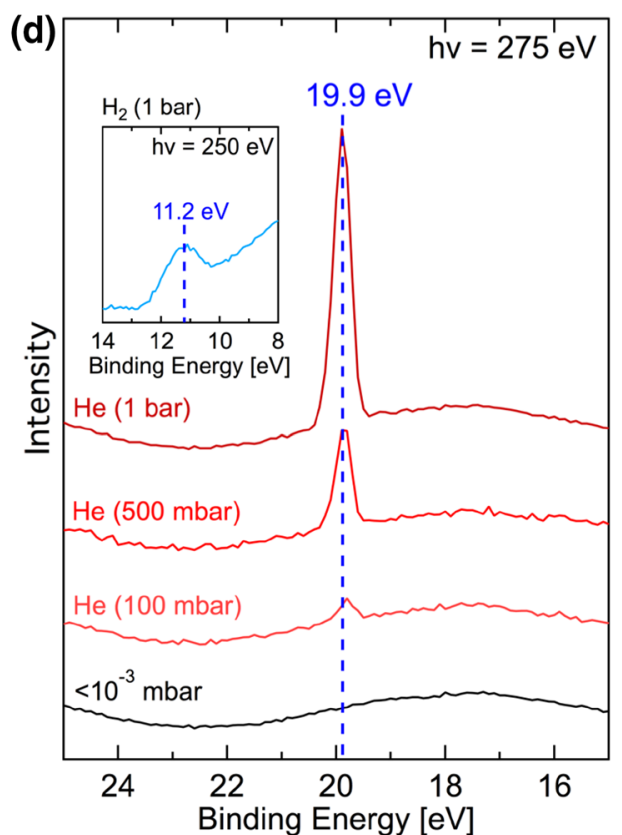

Fig. 6 a Schematic illustrating a single graphene covered pore within an array of many hundreds of holes that make up the membrane's active area. b O 1s XP spectra measured under vacuum $\left(<10^{-3} \mathrm{mbar}\right)$ and with $\mathrm{O}_{2}$ (1 bar), with a linear background subtracted. $\mathbf{c} \mathrm{Cu} 2 \mathrm{p}$ XP spectra SLG membrane covered with $\mathrm{Cu}(\sim 1 \mathrm{~nm})$ measured under vacuum $\left(<10^{-3} \mathrm{mbar}\right)$, first and second spectra measured in the presence of $\mathrm{O}_{2}(1 \mathrm{bar})$, measured again under vacuum $\left(<10^{-3} \mathrm{mbar}\right)$, and measured after $15 \mathrm{~min}$ of $\mathrm{O}_{2}(1 \mathrm{bar})$ exposure with the $\mathrm{X}$-ray

with time, and on returning to vacuum they rapidly reduce back to $\mathrm{Cu}^{+}$. The observed room temperature oxidation is seen to proceed more quickly when illuminated with the $\mathrm{X}$-ray beam (two lower red spectra in Fig. 6c), compared to if the beam is blanked for the first $15 \mathrm{~min}$ of $\mathrm{O}_{2}$ exposure (upper red spectrum in Fig. 6c), indicating it is at least partially induced by X-ray illumination, presumably as a result of radiolysis of the high pressure $\mathrm{O}_{2}$ to form ozone. The influence of the $\mathrm{X}$-ray beam is also seen when measuring graphene membranes with other atmospheric pressure gases behind them, where ongoing $\mathrm{X}$-ray beam irradiation is found to cause broadening of the $\mathrm{C} 1 \mathrm{~s}$ peak on the high-binding energy side, attributable to the creation of defects in the graphene and/or the accumulation of defective carbon [38]. This work also showed that given the high atomic density of atmospheric pressure gases, it is even possible to detect both $\mathrm{He}$ and $\mathrm{H}_{2}$ gases, which have traditionally been considered undetectable by XPS due to their low photoionization cross-sections. Figure $6 \mathrm{~d}$ shows that $\mathrm{He}$ is readily detectable at pressures of $100 \mathrm{mbar}$ and above, and that even $\mathrm{H}_{2}$ which has a 30 times lower photoionization cross-section, can be detected at 1 bar.

Velasco-Vélez et al. have also investigated atmospheric pressure reactions at solid-gas interfaces, using beam blanked. Shirley background is subtracted from all spectra. d He 1s XP spectra collected using a SLG membrane with the reaction cell under vacuum $\left(<10^{-3} \mathrm{mbar}\right)$, and filled with $\mathrm{He}(100 \mathrm{mbar}$, 500 mbar, and 1 bar). Inset: $H$ 1s spectra measured with the same membrane, with the reaction cell filled with $\mathrm{H}_{2}(1$ bar). No background subtraction is performed. Adapted with permission from [38]. Copyright 2016 American Chemical Society

PMMA-transferred bilayer graphene [100]. In this case they perform similar room temperature oxidation experiments with $\mathrm{Cu}$ nanoparticles, where they see very similar behaviour of oxidation towards $\mathrm{Cu}^{2+}$ in an atmosphere of $20 \% \mathrm{O}_{2}$ in $\mathrm{He}$ at 1 bar total pressure, whilst when this is exchanged for $5 \% \mathrm{H}_{2}$ in He they observe reduction back towards $\mathrm{Cu}^{+}$. These measurements were complicated somewhat by the presence of $\mathrm{Cu}$ contamination on the analyser side of the membranes, that may also have potentially been present between the graphene layers making up the bilayer, as a result of incomplete etching of the polycrystalline $\mathrm{Cu}$ foil on which the graphene was grown. To minimise the contribution from this contamination and maximise that coming from the $\mathrm{Cu}$ nanoparticles on the atmospheric pressure side, the XP spectra are acquired using photoelectrons with higher kinetic energies $(600 \mathrm{eV}$ rather than $200 \mathrm{eV})$. This higher kinetic energy may also account in part for a more intense $\mathrm{Cu} 2 \mathrm{p}$ signal, in spite of the thicker bilayer graphene membranes used. Possible $\mathrm{Cu}$ contamination was less of a problem for the measurements of Weatherup et al. as $\mathrm{Cu}$ nanoparticles were deposited onto the same side of the single-layer graphene as was originally in contact with the $\mathrm{Cu}$ growth catalyst, meaning lower kinetic energies could be 
used without erroneous contributions from contamination on the vacuum side.

Velasco-Vélez also investigated reactions occurring at elevated temperatures, which were achieved using direct laser heating of the membrane, similar to that illustrated in Fig. 4d. This includes the oxidation of Ir in an atmospheric pressure oxygen environment, as well as the study of a more industrially relevant reaction, namely the hydrogenation of propyne on Pd nanoparticles that had been drop-cast onto the graphene membrane. In this case changes in the $\mathrm{Pd} 3 \mathrm{~d}_{5 / 2}$ spectra were observed as the reaction environment was altered between gas mixtures of $\mathrm{He}, \mathrm{He} / \mathrm{H}_{2}$, and $\mathrm{He} / \mathrm{H}_{2} / \mathrm{C}_{3} \mathrm{H}_{4}$, indicating reduction of the initially oxidised $\mathrm{Pd}$ and then the uptake of C. In this work, the reaction environment was monitored using mass spectrometry and gas chromatography, which offer superior sensitivity compared to observing the gas-phase species with XPS, however they are not able to probe the reaction environment as locally.

Both of these reports on performing atmospheric pressure XPS of the solid-gas interface highlight the capability for performing spectroscopic measurements under realistic pressure and at elevated temperatures. Following these initial proof-of-concept measurements these techniques are expected to be applied more widely given their significant potential value to the broader catalysis community. As for the work to date on solid-liquid interfaces there have so far not been any reports that have successfully employed depth-profiling to reveal the variation in chemical environment close to the solid-gas interface but future studies may benefit from this.

\section{Outlook and Outstanding Challenges}

Despite the first demonstration of the 2D material membrane-based approach to atmospheric pressure XPS being reported more than 7 years ago [96], the number of groups to have successfully applied this method remains relatively small, with the experiments reported to date being overwhelmingly synchrotron-based. The primary reason for this appears to be the difficulty in producing leak-tight membranes that remain stable during measurement, and yet are thin enough and with large enough active area for a meaningful photoelectron signal to be obtained. The X-ray spot sizes used have been of $\sim 100 \mu \mathrm{m}$ in diameter or below, which given that the active area is typically of a similar size, is consistent with the significant challenge of avoiding defect/tears in the membranes as the size of the active area is increased further. For the 2D material membranebased approach to atmospheric pressure XPS to enter more widespread research use, requires extending this approach to laboratory-based XPS systems, given the greater availability of measurement systems and the lower cost of measurement time. This does not necessarily require a NAP-XPS system as the graphene membranes can maintain a large pressure difference between the reaction cell and analysis chamber, although protection of the analyser and X-ray source from any sudden spikes in pressure related to failure of the membranes should be carefully considered. The lower intensity and less focussed beam typically associated with lab-based $\mathrm{X}$-ray sources, mean that to avoid further sacrificing photoelectron signal a larger active region is required, increasing the challenge of reliable membrane manufacture. However, if this can be overcome the lower X-ray intensity and corresponding reduction in radiolytic attack of the membrane is likely to lead to improved stability and allow more extended measurements before failure occurs. Further improvements in the membrane fabrication process are key to this, in particular the transfer-step which is primarily responsible for introducing both mechanical damage and contamination. In this direction, completely eliminating the transfer step by directly growing graphene in place could avoid both of these issues. Although not trivial, current MEMS technology and the availability of low-temperature growth processes optimised for catalyst thin films, make this a realistic future prospect. This would enable the 2D material membranebased approach to atmospheric pressure photoelectron spectroscopy to move from the largely proof-of-concept experiments so far reported towards a technique that can be more broadly applied to the study of heterogeneous catalysis, where it promises important new insights into the surface chemistry occurring under realistic process conditions.

In addition to the powerful surface-sensitive chemical information that XPS can provide, in many experimental situations combining this with high spatial resolution would provide more profound understanding of the surface under investigation. A number of surface-sensitive spectromicroscopy techniques have already been used including scanning photoelectron microscopy (SPEM) [96], photoemission electron microscopy (PEEM) [98], and scanning AES [108], particularly with the multichannel static cell geometry, to identify which cells have and have not leaked. However, the resolution of these techniques is of the order of tens of nanometres, which is insufficient for revolving small nanoparticles let alone the atomic-scale structure of the surface, which is of primary interest in many cases. Therefore crosscorrelative studies using high-resolution imaging techniques such as transmission electron-microscopy (TEM) seem the most promising approach to achieve this. There has already been relatively widespread adoption of similar membranebased reaction cells within the EM research community, with multiple commercially available sample holders that make use of thin $\mathrm{Si}_{3} \mathrm{~N}_{4}$ membranes. The fact that similar cell geometries and materials can be used, make these techniques more readily comparable, and they may thus provide the information needed to decipher how the combination of 
surface structure and composition ultimately defines catalyst performance.

Although the membrane-based approach to operando XPS at atmospheric pressures remains in its infancy, we expect its use to expand significantly over the coming years with many groups starting to work in this area. Whilst applying this technique still involves a number of challenges, not least the fabrication of 2D material membranes, overcoming these offers the prospect of accessing nanometre-scale, depth-resolved chemical information about both solid-liquid and solid-gas interfaces under conditions where this has never before been possible. We therefore envisage this becoming a valuable technique for improving our understanding of the chemistry that occurs in industrial catalytic reactions, as well as providing a valuable counterpart to high-resolution spatially resolved studies.

Acknowledgements R.S.W. acknowledges a Research Fellowship from St. John's College, Cambridge and a EU Marie Skłodowska-Curie Individual Fellowship (Global) under grant ARTIST (No. 656870) from the European Union's Horizon 2020 research and innovation programme.

Open Access This article is distributed under the terms of the Creative Commons Attribution 4.0 International License (http://creativeco mmons.org/licenses/by/4.0/), which permits unrestricted use, distribution, and reproduction in any medium, provided you give appropriate credit to the original author(s) and the source, provide a link to the Creative Commons license, and indicate if changes were made.

\section{References}

1. Schlögl R (2015) Heterogeneous catalysis. Angew Chem 54:3465-3520. https://doi.org/10.1002/anie.201410738

2. Siegbahn K, Nordling C, Johansson G et al (1969) ESCA applied to free molecules. North-Holland, Amsterdam

3. Salmeron M, Schlögl R (2008) Ambient pressure photoelectron spectroscopy: a new tool for surface science and nanotechnology. Surf Sci Rep 63:169-199. https://doi.org/10.1016/j.surfr ep.2008.01.001

4. Ogletree DF, Bluhm H, Lebedev G et al (2002) A differentially pumped electrostatic lens system for photoemission studies in the millibar range. Rev Sci Instrum. https://doi.org/10.1063/1.15123 36

5. Wu CH, Weatherup RS, Salmeron MB (2015) Probing electrode/ electrolyte interfaces in situ by X-ray spectroscopies: old methods, new tricks. Phys Chem Chem Phys 17:30229-30239. https ://doi.org/10.1039/C5CP04058B

6. Axnanda S, Crumlin EJ, Mao B et al (2015) Using "Tender" $\mathrm{X}$-ray ambient pressure X-ray photoelectron spectroscopy as a direct probe of solid-liquid interface. Sci Rep. https://doi. org/10.1038/srep09788

7. Karslıoğlu O, Nemšák S, Zegkinoglou I et al (2015) Aqueous solution/metal interfaces investigated in operando by photoelectron spectroscopy. Faraday Discuss 180:35-53. https://doi. org/10.1039/C5FD00003C

8. de Jonge N, Ross FM (2011) Electron microscopy of specimens in liquid. Nat Nanotechnol 6:695-704. https://doi.org/10.1038/ nnano.2011.161
9. Jensen E, Mølhave K (2017) Encapsulated liquid cells for transmission electron microscopy. In: Ross FM (ed) Liquid cell electron microscopy. Cambridge University Press, Cambridge, pp 35-55

10. Jiang P, Chen J-L, Borondics F et al (2010) In situ soft X-ray absorption spectroscopy investigation of electrochemical corrosion of copper in aqueous $\mathrm{NaHCO}_{3}$ solution. Electrochem commun 12:820-822. https://doi.org/10.1016/j.elecom.2010.03.042

11. Velasco-Velez J-J, Wu CH, Pascal TA et al (2014) The structure of interfacial water on gold electrodes studied by X-ray absorption spectroscopy. Science 346:831-834

12. Escudero C, Salmeron M (2013) From solid-vacuum to solidgas and solid-liquid interfaces: in situ studies of structure and dynamics under relevant conditions. Surf Sci 607:2-9. https:// doi.org/10.1016/j.susc.2012.08.007

13. Weatherup RS, Wu CH, Escudero C et al (2018) Environment-dependent radiation damage in atmospheric pressure X-ray spectroscopy. J Phys Chem B 122:737-744. https://doi. org/10.1021/acs.jpcb.7b06397

14. Joly L, Otero E, Choueikani F et al (2014) Fast continuous energy scan with dynamic coupling of the monochromator and undulator at the DEIMOS beamline. J Synchrotron Radiat 21:502-506. https://doi.org/10.1107/S1600577514003671

15. Frahm R (1989) New method for time dependent X-ray absorption studies. Rev Sci Instrum 60:2515-2518. https://doi. org/10.1063/1.1140716

16. Kratzer M, Bayer BC, Kidambi PR et al (2015) Effects of polymethylmethacrylate-transfer residues on the growth of organic semiconductor molecules on chemical vapor deposited graphene. Appl Phys Lett. https://doi.org/10.1063/1.4913948

17. Tanuma S, Powell CJ, Penn DR (2011) Calculations of electron inelastic mean free paths. IX. Data for 41 elemental solids over the $50 \mathrm{eV}$ to $30 \mathrm{keV}$ range. Surf Interface Anal 43:689-713. https://doi.org/10.1002/sia.3522

18. Tanuma S, Powell CJ, Penn DR (1994) Calculations of electron inelastic mean free paths. V. Data for 14 organic compounds over the 50-2000 eV range. Surf Interface Anal 21:165-176. https://doi.org/10.1002/sia.740210302

19. Seah MP, Dench WA (1979) Quantitative electron spectroscopy of surfaces. Surf Interface Anal 1:2-11

20. Werner WSM (2001) Electron transport in solids for quantitative surface analysis. Surf Interface Anal 31:141-176. https:// doi.org/10.1002/sia.973

21. Masuda T, Yoshikawa H, Noguchi H et al (2013) In situ $\mathrm{X}$-ray photoelectron spectroscopy for electrochemical reactions in ordinary solvents. Appl Phys Lett. https://doi. org/10.1063/1.4821180

22. Yeh JJ, Lindau I (1985) Atomic subshell photoionization cross sections and asymmetry parameters: $1 \leq \mathrm{Z} \leq 103$. At Data Nucl Data Tables 32:1-55

23. Bacon GE (1951) The interlayer spacing of graphite. Acta Crystallogr 4:558-561. https://doi.org/10.1107/S0365110X5 1001781

24. Bunch JS, Verbridge SS, Alden JS et al (2008) Impermeable atomic membranes from graphene sheets. Nano Lett 8:24582462. https://doi.org/10.1021/n1801457b

25. Dlubak B, Martin M-B, Weatherup RS et al (2012) Graphenepassivated nickel as an oxidation-resistant electrode for spintronics. ACS Nano 6:10930-10934. https://doi.org/10.1021/nn304 $424 \mathrm{x}$

26. Martin M-B, Dlubak B, Weatherup RS et al (2014) Sub-nanometer atomic layer deposition for spintronics in magnetic tunnel junctions based on graphene spin-filtering membranes. ACS Nano 8:7890-7895. https://doi.org/10.1021/nn5017549 
27. Martin M-B, Dlubak B, Weatherup RS et al (2015) Protecting nickel with graphene spin-filtering membranes: a single layer is enough. Appl Phys Lett. https://doi.org/10.1063/1.4923401

28. Piquemal-Banci M, Galceran R, Caneva S et al (2016) Magnetic tunnel junctions with monolayer hexagonal boron nitride tunnel barriers. Appl Phys Lett. https://doi.org/10.1063/1.4943516

29. Weatherup RS, D'Arsié L, Cabrero-Vilatela A et al (2015) Longterm passivation of strongly interacting metals with single-layer graphene. J Am Chem Soc 137:14358-14366. https://doi. org/10.1021/jacs.5b08729

30. Walker MI, Braeuninger-Weimar P, Weatherup RS et al (2015) Measuring the proton selectivity of graphene membranes. Appl Phys Lett. https://doi.org/10.1063/1.4936335

31. Walker MI, Ubych K, Saraswat V et al (2017) Extrinsic behaviour of 2D membranes for ionic selectivity. ACS Nano 11:13401346. https://doi.org/10.1021/acsnano.6b06034

32. Schriver M, Regan W, Gannett WJ et al (2013) Graphene as a long-term metal oxidation barrier: worse than nothing. ACS Nano 7:5763-5768. https://doi.org/10.1021/nn4014356

33. Weatherup RS, Dlubak B, Hofmann S (2012) Kinetic control of catalytic CVD for high quality graphene at low temperatures. ACS Nano 6:9996-10003. https://doi.org/10.1021/nn303674g doi

34. Lee C, Wei X, Kysar JW, Hone J (2008) Measurement of the elastic properties and intrinsic strength of monolayer graphene. Science 321:385-388. https://doi.org/10.1126/science.1157996

35. Rasool HI, Ophus C, Klug WS et al (2013) Measurement of the intrinsic strength of crystalline and polycrystalline graphene. Nat Commun. https://doi.org/10.1038/ncomms3811

36. Lee G-H, Cooper RC, An SJ et al (2013) High-strength chemical-vapor-deposited graphene and grain boundaries. Science 340:1073-1076. https://doi.org/10.1126/science.1235126

37. Zandiatashbar A, Lee G-H, An SJ et al (2014) Effect of defects on the intrinsic strength and stiffness of graphene. Nat Commun 5:1-9. https://doi.org/10.1038/ncomms4186

38. Weatherup RS, Eren B, Hao Y et al (2016) Graphene membranes for atmospheric pressure photoelectron spectroscopy. J Phys Chem Lett 7:1622-1627. https://doi.org/10.1021/acs.jpcle tt.6b00640

39. Wang L, Williams CM, Boutilier MSH et al (2017) Single-layer graphene membranes withstand ultrahigh applied pressure. Nano Lett 17:3081-3088. https://doi.org/10.1021/acs.nanolett.7b00442

40. Colón Santana JA (2015) Charging effects in X-ray photoelectron spectroscopy. In: Quantitative core level photoelectron spectroscopy. Morgan \& Claypool Publishers, San Rafael, pp 1-11

41. Vanýsek P, Elec-VKTS (2016) Electrochemical series. CRC Handb Chem Phys, 97th edn, Sect. 5 Thermo, Electro Solut Chem 5:78-84. https://doi.org/10.1136/oem.53.7.504

42. Li LH, Cervenka J, Watanabe K et al (2014) Strong oxidation resistance of atomically thin boron nitride nanosheets. ACS Nano 8:1457-1462. https://doi.org/10.1021/nn500059s

43. Novoselov KS, Geim AK, Morozov SV et al (2004) Electric field effect in atomically thin carbon films. Science 306:666-669. https://doi.org/10.1126/science.1102896

44. Hernandez Y, Nicolosi V, Lotya M et al (2008) High-yield production of graphene by liquid-phase exfoliation of graphite. Nat Nanotechnol 3:563-568. https://doi.org/10.1038/nnano.2008.215

45. Paton KR, Varrla E, Backes C et al (2014) Scalable production of large quantities of defect-free few-layer graphene by shear exfoliation in liquids. Nat Mater 13:624-630. https://doi.org/10.1038/ nmat3944

46. Li D, Müller MB, Gilje S et al (2008) Processable aqueous dispersions of graphene nanosheets. Nat Nanotechnol 3:101-105. https://doi.org/10.1038/nnano.2007.451
47. Eda G, Fanchini G, Chhowalla M (2008) Large-area ultrathin films of reduced graphene oxide as a transparent and flexible electronic material. Nat Nanotechnol 3:270-274. https://doi. org/10.1038/nnano.2008.83

48. Berger C, Song Z, Li T et al (2004) Ultrathin epitaxial graphite: $2 \mathrm{D}$ electron gas properties and a route toward graphene-based nanoelectronics. J Phys Chem B 108:19912-19916. https://doi. org/10.1021/jp040650f

49. Emtsev KV, Bostwick A, Horn K et al (2009) Towards wafer-size graphene layers by atmospheric pressure graphitization of silicon carbide. Nat Mater 8:203-207. https://doi.org/10.1038/nmat2382

50. Unarunotai S, Murata Y, Chialvo CE et al (2009) Transfer of graphene layers grown on $\mathrm{SiC}$ wafers to other substrates and their integration into field effect transistors. Appl Phys Lett. https:// doi.org/10.1063/1.3263942

51. Kim J, Park H, Hannon JB, Bedell SW, Fogel K, Devendra K. Sadana CD (2014) Layer-resolved graphene transfer via engineered strain layers. Science 342:833-837. https://doi. org/10.1126/science.1242988

52. Novoselov KS, Fal'ko VI, Colombo L et al (2012) A roadmap for graphene. Nature 490:192-200. https://doi.org/10.1038/natur e11458

53. Hofmann S, Braeuninger-Weimer P, Weatherup RS (2015) CVDenabled graphene manufacture and technology. J Phys Chem Lett 6:2714-2721. https://doi.org/10.1021/acs.jpclett.5b01052

54. Huang PY, Ruiz-Vargas CS, van der Zande AM et al (2011) Grains and grain boundaries in single-layer graphene atomic patchwork quilts. Nature 469:389-392. https://doi.org/10.1038/ nature09718

55. Weatherup RS, Shahani AJ, Wang Z-J et al (2016) In situ graphene growth dynamics on polycrystalline catalyst foils. Nano Lett 16:6196-6206. https://doi.org/10.1021/acs.nanolett.6b024 59

56. Eizenberg M, Blakely J (1979) Carbon monolayer phase condensation on Ni (111). Surf Sci 82:228-236

57. Oshima C, Nagashima A (1997) Ultra-thin epitaxial films of graphite and hexagonal boron nitride on solid surfaces. J Phys Condens Matter 9:1-20

58. Li X, Cai W, An J et al (2009) Large-area synthesis of highquality and uniform graphene films on copper foils. Science 324:1312-1314. https://doi.org/10.1126/science.1171245

59. Li X, Cai W, Colombo L, Ruoff RS (2009) Evolution of graphene growth on $\mathrm{Ni}$ and $\mathrm{Cu}$ by carbon isotope labeling. Nano Lett 9:4268-4272

60. Reina A, Jia X, Ho J et al (2009) Large area, few-layer graphene films on arbitrary substrates by chemical vapor deposition. Nano Lett 9:30-35. https://doi.org/10.1021/n1801827v

61. Reina A, Thiele S, Jia X et al (2009) Growth of large-area single- and bi-layer graphene by controlled carbon precipitation on polycrystalline Ni surfaces. Nano Res 9:30-35. https://doi. org/10.1007/s12274-009-9059-y

62. Mattevi C, Kim H, Chhowalla M (2011) A review of chemical vapour deposition of graphene on copper. J Mater Chem. https ://doi.org/10.1039/c0jm02126a

63. Weatherup RS, Bayer BC, Blume R et al (2011) In situ characterization of alloy catalysts for low-temperature graphene growth. Nano Lett 11:4154-4160. https://doi.org/10.1021/nl202036y

64. Weatherup RS, Bayer BC, Blume R et al (2012) On the mechanisms of Ni-catalysed graphene chemical vapour deposition. ChemPhysChem 13:2544-2549. https://doi.org/10.1002/ cphc. 201101020

65. Cabrero-Vilatela A, Weatherup RS, Braeuninger-Weimer P et al (2016) Towards a general growth model for graphene CVD on transition metal catalysts. Nanoscale 8:2149-2158. https://doi. org/10.1039/C5NR06873H 
66. Gao L, Ren W, Xu H et al (2012) Repeated growth and bubbling transfer of graphene with millimetre-size single-crystal grains using platinum. Nat Commun. https://doi.org/10.1038/ncomm s1702

67. Sutter E, Albrecht P, Sutter P (2009) Graphene growth on polycrystalline Ru thin films. Appl Phys Lett. https://doi. org/10.1063/1.3224913

68. Kidambi PR, Ducati C, Dlubak B et al (2012) The parameter space of graphene chemical vapor deposition on polycrystalline Cu. J Phys Chem C 116:22492-22501. https://doi.org/10.1021/ jp303597m

69. Hao Y, Bharathi MS, Wang L et al (2013) The role of surface oxygen in the growth of large single-crystal graphene on copper. Science 342:720-723. https://doi.org/10.1126/scien ce. 1243879

70. Sugime H, D'Arsiè L, Esconjauregui S et al (2017) Low temperature growth of fully covered single-layer graphene using $\mathrm{CoCu}$ catalyst. Nanoscale. https://doi.org/10.1039/C7NR02553J

71. Yu Q, Jauregui L, Wu W et al (2011) Control and characterization of individual grains and grain boundaries in graphene grown by chemical vapour deposition. Nat Mater 10:443-449. https:// doi.org/10.1038/nmat3010

72. Caneva S, Weatherup RS, Bayer B et al (2015) Nucleation control for large, single crystalline domains of monolayer hexagonal boron nitride via Si-doped Fe catalysts. Nano Lett 15:1867-1875. https://doi.org/10.1021/n15046632

73. Wu T, Zhang X, Yuan Q et al (2016) Fast growth of inch-sized single-crystalline graphene from a controlled single nucleus on $\mathrm{Cu}-\mathrm{Ni}$ alloys. Nat Mater 15:43-47. https://doi.org/10.1038/ nmat 4477

74. Lee J-H, Lee EK, Joo W-J et al (2014) Wafer-scale growth of single-crystal monolayer graphene on reusable hydrogen-terminated germanium. Science 344:286-289. https://doi.org/10.1126/scien ce. 1252268

75. Xu X, Zhang Z, Dong J et al (2017) Ultrafast epitaxial growth of metre-sized single-crystal graphene on industrial $\mathrm{Cu}$ foil. Sci Bull 62:1074-1080. https://doi.org/10.1016/j.scib.2017.07.005

76. Wang Y, Zheng Y, Xu X et al (2011) Electrochemical delamination of CVD-grown graphene film: toward the recyclable use of copper catalyst. ACS Nano 5:9927-9933. https://doi. org/10.1021/nn203700w

77. Wang X, Dolocan A, Chou H et al (2017) Direct observation of poly(methyl methacrylate) removal from a graphene surface. Chem Mater 29:2033-2039. https://doi.org/10.1021/acs.chemm ater.6b03875

78. Ahn Y, Kim J, Ganorkar S et al (2016) Thermal annealing of graphene to remove polymer residues. Mater Express 6:69-76. https://doi.org/10.1166/mex.2016.1272

79. Lin W-H, Chen T-H, Chang J-K et al (2014) Direct and polymerfree method for transferring graphene grown by chemical vapor deposition to any substrate. ACS Nano 8:1784-1791. https://doi. org/10.1021/nn406170d

80. Zhang G, Güell AG, Kirkman PM et al (2016) Versatile polymerfree graphene transfer method and applications. ACS Appl Mater Interfaces 8:8008-8016. https://doi.org/10.1021/acsami.6b00681

81. Aria AI, Kidambi PR, Weatherup RS et al (2016) Time evolution of the wettability of supported graphene under ambient air exposure. J Phys Chem C 120:2215-2224. https://doi.org/10.1021/ acs.jpcc.5b10492

82. Li Z, Wang Y, Kozbial A et al (2013) Effect of airborne contaminants on the wettability of supported graphene and graphite. Nat Mater 12:925-931. https://doi.org/10.1038/nmat3709

83. Kim HH, Lee SK, Lee SG et al (2016) Wetting-assisted crackand wrinkle-free transfer of wafer-scale graphene onto arbitrary substrates over a wide range of surface energies. Adv Funct Mater 26:2070-2077. https://doi.org/10.1002/adfm.201504551
84. John R, Vig (1985) UV/ozone cleaning of surfaces. J Vac Sci Technol A 3:1027-1034

85. Tong Q-Y, Lee T-H, Gösele U et al (1997) The role of surface chemistry in bonding of standard silicon wafers. J Electrochem Soc. https://doi.org/10.1149/1.1837415

86. Alam AU, Howlader MMR, Deen MJ (2014) The effects of oxygen plasma and humidity on surface roughness, water contact angle and hardness of silicon, silicon dioxide and glass. J Micromech Microeng. https://doi.org/10.1088/0960-1317/24/3/035010

87. Yoon T, Shin WC, Kim TY et al (2012) Direct measurement of adhesion energy of monolayer graphene as-grown on copper and its application to renewable transfer process. Nano Lett 12:1448-1452. https://doi.org/10.1021/nl204123h

88. Lock EH, Baraket M, Laskoski M et al (2012) High-quality uniform dry transfer of graphene to polymers. Nano Lett 12:102107. https://doi.org/10.1021/nl203058s

89. Fechine GJM, Martin-Fernandez I, Yiapanis G et al (2015) Direct dry transfer of chemical vapor deposition graphene to polymeric substrates. Carbon 83:224-231. https://doi.org/10.1016/j.carbo n.2014.11.038

90. Mueller NS, Morfa AJ, Abou-Ras D et al (2014) Growing graphene on polycrystalline copper foils by ultra-high vacuum chemical vapor deposition. Carbon 78:347-355. https://doi. org/10.1016/j.carbon.2014.07.011

91. Luo Z, Lu Y, Singer DW et al (2011) Effect of substrate roughness and feedstock concentration on growth of wafer-scale graphene at atmospheric pressure. Chem Mater 23:1441-1447. https ://doi.org/10.1021/cm1028854

92. Hattab H, N'Diaye AT, Wall D et al (2012) Interplay of wrinkles, strain, and lattice parameter in graphene on iridium. Nano Lett 12:678-682. https://doi.org/10.1021/nl203530t

93. Liu N, Pan Z, Fu L et al (2011) The origin of wrinkles on transferred graphene. Nano Res 4:996-1004. https://doi.org/10.1007/ s12274-011-0156-3

94. Choi J, Kwak J, Park S et al (2014) Growth of wrinkle-free graphene on texture-controlled platinum films and thermal-assisted transfer of large-scale patterned graphene. ACS Nano 9:679-686

95. Deng B, Pang Z, Chen S et al (2017) Wrinkle-free single-crystal graphene wafer grown on strain-engineered substrates. ACS Nano. https://doi.org/10.1021/acsnano.7b06196

96. Kolmakov A, Dikin DA, Cote LJ et al (2011) Graphene oxide windows for in situ environmental cell photoelectron spectroscopy. Nat Nanotechnol 6:651-657. https://doi.org/10.1038/nnano 2011.130

97. Kraus J, Reichelt R, Günther S et al (2014) Photoelectron spectroscopy of wet and gaseous samples through graphene membranes. Nanoscale 6:14394-14403. https://doi.org/10.1039/ C4NR03561E

98. Guo H, Strelcov E, Yulaev A et al (2017) Enabling photoemission electron microscopy in liquids via graphene-capped microchannel arrays. Nano Lett. https://doi.org/10.1021/acs.nanol ett.6b04460

99. Velasco-Velez JJ, Pfeifer V, Hävecker M et al (2015) Photoelectron spectroscopy at the graphene-liquid interface reveals the electronic structure of an electrodeposited cobalt/graphene electrocatalyst. Angew Chem Int Ed 54:14554-14558. https://doi. org/10.1002/anie.201506044

100. Velasco-Vélez JJ, Pfeifer V, Hävecker M et al (2016) Atmospheric pressure X-ray photoelectron spectroscopy apparatus: bridging the pressure gap. Rev Sci Instrum. https://doi. org/10.1063/1.4951724

101. Machado BF, Serp P (2012) Graphene-based materials for catalysis. Catal Sci Technol 2:54-75. https://doi.org/10.1039/C1CY0 0361E 
102. Julkapli NM, Bagheri S (2015) Graphene supported heterogeneous catalysts: an overview. Int J Hydrog Energy 40:948-979. https://doi.org/10.1016/j.ijhydene.2014.10.129

103. Novoselov KS, Mishchenko A, Carvalho A, Castro Neto AH (2016) 2D materials and van der Waals heterostructures. Science. https://doi.org/10.1126/science.aac9439

104. Dlubak B, Kidambi PR, Weatherup RS et al (2012) Substrateassisted nucleation of ultra-thin dielectric layers on graphene by atomic layer deposition. Appl Phys Lett. https://doi. org/10.1063/1.4707376

105. Dlubak B, Martin M-B, Deranlot C et al (2012) Homogeneous pinhole free $1 \mathrm{~nm} \mathrm{Al}_{2} \mathrm{O}_{3}$ tunnel barriers on graphene. Appl Phys Lett. https://doi.org/10.1063/1.4765348

106. Caneva S, Martin MB, D’Arsié L et al (2017) From growth surface to device interface: preserving metallic Fe under monolayer hexagonal boron nitride. ACS Appl Mater Interfaces 9:2997329981. https://doi.org/10.1021/acsami.7b08717

107. Kidambi PR, Blume R, Kling J et al (2014) In situ observations during chemical vapor deposition of hexagonal boron nitride on polycrystalline copper. Chem Mater 26:6380-6392. https://doi. org/10.1021/cm502603n

108. Yulaev A, Guo H, Strelcov E et al (2017) Graphene microcapsule arrays for combinatorial electron microscopy and spectroscopy in liquids. ACS Appl Mater Interfaces 9:26492-26502. https:// doi.org/10.1021/acsami.7b02824

109. Blume R, Kidambi PR, Bayer BC et al (2014) The influence of intercalated oxygen on the properties of graphene on polycrystalline $\mathrm{Cu}$ under various environmental conditions. Phys Chem Chem Phys 16:25989-26003. https://doi.org/10.1039/C4CP0 4025B

110. Kidambi PR, Bayer BC, Blume R et al (2013) Observing graphene grow: catalyst-graphene interactions during scalable graphene growth on polycrystalline copper. Nano Lett 13:47694778. https://doi.org/10.1021/nl4023572

111. Shikin AM, Adamchuk VK, Rieder K-H (2009) Formation of quasi-free graphene on the $\mathrm{Ni}(111)$ surface with intercalated $\mathrm{Cu}$, Ag, and Au layers. Phys Solid State 51:2390-2400. https://doi. org/10.1134/S1063783409110316

112. Walter AL, Nie S, Bostwick A et al (2011) Electronic structure of graphene on single-crystal copper substrates. Phys Rev B 84:1-7. https://doi.org/10.1103/PhysRevB.84.195443

113. Wilson NR, Marsden AJ, Saghir M et al (2013) Weak mismatch epitaxy and structural feedback in graphene growth on copper foil. Nano Res 6:99-112. https://doi.org/10.1007/s1227 4-013-0285-y

114. Nie S, Walter AL, Bartelt NC et al (2011) Growth from below: graphene bilayers on $\operatorname{Ir}(111)$. ACS Nano 5:2298-2306. https:// doi.org/10.1021/nn103582g

115. Sutter P, Sadowski JT, Sutter E (2009) Graphene on Pt (111): growth and substrate interaction. Phys Rev B. https://doi. org/10.1103/PhysRevB.80.245411

116. Dahal A, Batzill M (2014) Graphene-nickel interfaces: a review. Nanoscale 6:2548-2562. https://doi.org/10.1039/c3nr05279f

117. Shikin A, Prudnikova G, Adamchuk V et al (2000) Surface intercalation of gold underneath a graphite monolayer on $\mathrm{Ni}(111)$ studied by angle-resolved photoemission and high-resolution electron-energy-loss spectroscopy. Phys Rev B 62:13202-13208. https://doi.org/10.1103/PhysRevB.62.13202

118. Weatherup RS, Amara H, Blume R et al (2014) Interdependency of subsurface carbon distribution and graphene-catalyst interactions. J Am Chem Soc 136:13698-13708

119. Nagashima A, Tejima N, Oshima C (1994) Electronic states of the pristine and alkali-metal-intercalated monolayer graphite/Ni(111) systems. Phys Rev B 50:17487-17495. https://doi. org/10.1103/PhysRevB.50.17487
120. Varykhalov A, Sánchez-Barriga J, Shikin A et al (2008) Electronic and magnetic properties of quasifreestanding graphene on Ni. Phys Rev Lett. https://doi.org/10.1103/PhysRevLet t.101.157601

121. Voloshina E, Dedkov Y (2012) Graphene on metallic surfaces: problems and perspectives. Phys Chem Chem Phys 14:1350213514. https://doi.org/10.1039/c2cp42171b

122. Eom D, Prezzi D, Rim KT et al (2009) Structure and electronic properties of graphene nanoislands on Co(0001). Nano Lett 9:2844-2848. https://doi.org/10.1021/n1900927f

123. Varykhalov A, Rader O (2009) Graphene grown on Co(0001) films and islands: electronic structure and its precise magnetization dependence. Phys Rev B. https://doi.org/10.1103/PhysR evB.80.035437

124. Vinogradov NA, Zakharov AA, Kocevski V et al (2012) Formation and structure of graphene waves on Fe(110). Phys Rev Lett. https://doi.org/10.1103/PhysRevLett.109.026101

125. Walker MI, Weatherup RS, Bell NAW et al (2015) Free-standing graphene membranes on glass nanopores for ionic current measurements. Appl Phys Lett 106:23119

126. O'Hern SC, Boutilier MSH, Idrobo JC et al (2014) Selective ionic transport through tunable subnanometer pores in singlelayer graphene membranes. Nano Lett 14:1234-1241. https:// doi.org/10.1021/n1404118f

127. Tracz A, Wegner G, Rabe JP (1993) Kinetics of surface roughening via pit growth during the oxidation of the basal plane of graphite. 1. Experiments. Langmuir 9:3033-3038. https://doi. org/10.1021/la00035a048

128. Tracz A, Kalachev AA, Wegner G, Rabe JP (1995) Control over nanopits on the basal plane of graphite by remote argon plasma and subsequent thermal oxidation. Langmuir 11:2840-2842. https://doi.org/10.1021/la00007a080

129. Nan HY, Ni ZH, Wang J et al (2013) The thermal stability of graphene in air investigated by Raman spectroscopy. J Raman Spectrosc 44:1018-1021. https://doi.org/10.1002/jrs.4312

130. Eberhardt W, Sham TK, Carr R et al (1983) Site-specific fragmentation of small molecules following soft-X-ray excitation. Phys Rev Lett 50:1038-1041. https://doi.org/10.1103/PhysR evLett.50.1038

131. Nenner I, Morin P (1996) Electronic and nuclear relaxation of core-excited molecules. In: Becker U, Shirley DA (eds) VUV and soft X-ray photoionization. Plenum Press, New York, pp 291-354

132. Stoll JD, Kolmakov A (2012) Electron transparent graphene windows for environmental scanning electron microscopy in liquids and dense gases. Nanotechnology. https://doi.org/10.1088/09574484/23/50/505704

133. Nemšák SS, Strelcov E, Duchoň T et al (2017) Interfacial electrochemistry in liquids probed with photoemission electron microscopy. J Am Chem Soc 139:18138-18141. https://doi. org/10.1021/jacs.7b07365

134. Liu Z, Gong Y, Zhou W et al (2013) Ultrathin high-temperature oxidation-resistant coatings of hexagonal boron nitride. Nat Commun. https://doi.org/10.1038/ncomms3541

135. Wang H, Wu CH, Weatherup RS et al (2018) X-ray-induced fragmentation of imidazolium-based ionic liquids studied by soft X-ray absorption spectroscopy. J Phys Chem Lett. https://doi. org/10.1021/acs.jpclett.8b00057

136. Cho H, Jones MR, Nguyen SC et al (2017) The use of graphene and its derivatives for liquid-phase transmission electron microscopy of radiation-sensitive specimens. Nano Lett 17:414-420. https://doi.org/10.1021/acs.nanolett.6b04383 